Soto, H., Schurr, B. (2021): DeepPhasePick: A method for detecting and picking seismic phases from local earthquakes based on highly optimized convolutional and recurrent deep neural networks. - Geophysical Journal International, 227, 2, 1268-1294.

https://doi.org/10.1093/gji/ggab266 


\title{
DeepPhasePick: a method for detecting and picking seismic phases from local earthquakes based on highly optimized convolutional and recurrent deep neural networks
}

\author{
Hugo Soto ${ }^{\odot}$ and Bernd Schurr \\ German Research Centre for Geosciences (GFZ), Potsdam, Germany.E-mail: soto@gfz-potsdam.de
}

Received 2021 June 26; in original form 2020 October 2

\begin{abstract}
SUMMAR Y
Seismic phase detection, identification and first-onset picking are basic but essential routines to analyse earthquake data. As both the number of seismic stations, globally and regionally, and the number of experiments greatly increase due to ever greater availability of instrumentation, automated data processing becomes more and more essential. For example for modern seismic experiments involving 100 s to even 1000s instruments, conventional human analyst-based identification and picking of seismic phases is becoming unfeasible, and the introduction of automatic algorithms mandatory. In this paper, we introduce DeepPhasePick, an automatic two-stage method that detects and picks $P$ and $S$ seismic phases from local earthquakes. The method is entirely based on highly optimized deep neural networks, consisting of a first stage that detects the phases using a convolutional neural network, and a second stage that uses two recurrent neural networks to pick both phases. Detection is performed on threecomponent seismograms. $P$ - and $S$-picking is then conducted on the vertical and the twohorizontal components, respectively. Systematic hyperparameter optimization was applied to select the best model architectures and to define both the filter applied to pre-process the seismic data as well as the characteristics of the window sample used to feed the models. We trained DeepPhasePick using seismic records extracted from two sets of manually picked event waveforms originating from northern Chile ( $\sim 39000$ records for detection and $\sim 36000$ records for picking). In different tectonic regimes, DeepPhasePick demonstrated the ability to both detect $P$ and $S$ phases from local earthquakes with high accuracy, as well as predict $P$ - and $S$-phase time onsets with an analyst level of precision. DeepPhasePick additionally computes onset uncertainties based on the Monte Carlo Dropout technique as an approximation of Bayesian inference. This information can then further feed an associator algorithm in an earthquake location procedure.
\end{abstract}

Key words: South America; Neural networks, fuzzy logic; Time-series analysis; Body waves; Computational seismology.

\section{INTRODUCTION}

One of the most fundamental components in any earthquake hypocentre estimation routine is the identification and picking of the arriving $P$ and $S$ seismic phases. The identification of the $S$ phase arrivals is especially important, since this helps to better constrain hypocentre depths obtained only from $P$-phase arrivals. In the past, the phase picking task was commonly performed manually by analysts, who identified each phase arrival based on their training and experience. However, as the available seismic data has rapidly increased over time, the use of automatic phase detection algorithms has become increasingly necessary.
These automatic algorithms encompass detectors which are based on the energy or frequency content of the seismic waveforms such as STA/LTA (e.g. Allen 1978; Baer \& Kradolfer 1987; Earle \& Shearer 1994; Sleeman \& van Eck 1999; Aldersons 2004; Di Stefano et al. 2006; Diehl et al. 2009), those based on correlations of template waveforms against continuous seismic data (e.g. Van Trees 1968; Harris 1991; Gibbons \& Ringdal 2006), and detectors based on the representation of seismic data as a linear combination of orthogonal basis waveforms (Scharf \& Friedlander 1994; Harris 1997, 2001).

Phase detectors based on frequency or energy have been used in the past as part of multistage automatic earthquake location procedures that allowed the creation of high-quality earthquake 


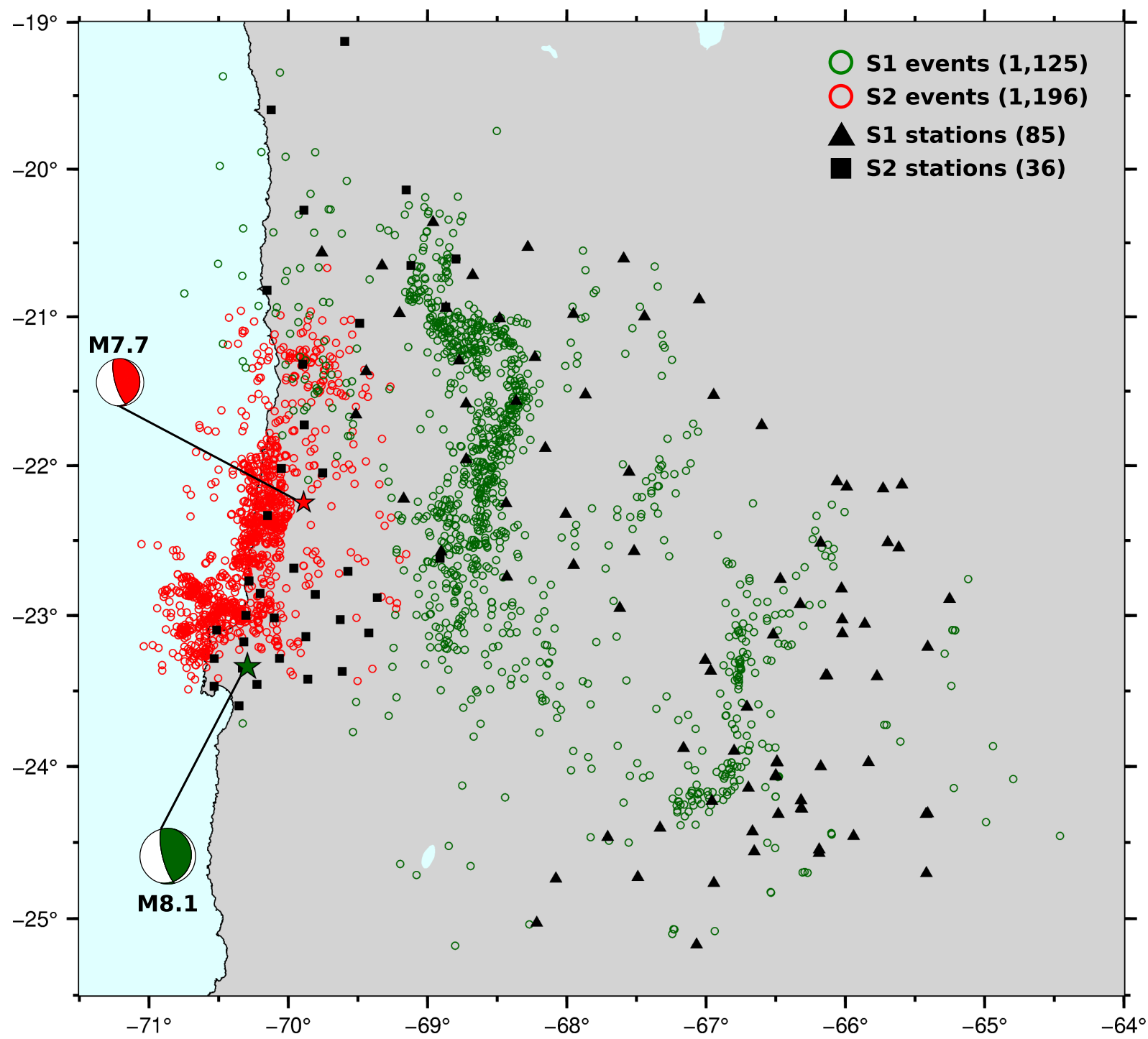

Figure 1. Events data sets from which window samples picked by analysts were extracted for training the phase detection and picking models in DeepPhasePick. Events in S1 and S2 data sets are plotted as green and red circles, respectively. Focal mechanisms (beachballs) and epicentres (stars) of the 1995-07-30 M8.1 Antofagasta and the 2007-11-14 M7.7 Tocopilla earthquakes are plotted in green and red, for reference. Window samples used in this work come from the picked stations plotted as black triangles (from S1) and squares (from S2).

catalogues, for example for the Northern Chile region (Sippl et al. 2018; Soto et al. 2019). Correlation detectors, such as the matched filter method (Van Trees 1968), rely on the similarity of known template waveforms and have been widely used for detecting repeating earthquakes (e.g. Nadeau \& Johnson 1998; Igarashi et al. 2003; Kato \& Igarashi 2012; Huang et al. 2017; Folesky et al. 2018) or searching for missing events (e.g. Shelly et al. 2007; Peng \& Zhao 2009; Ross et al. 2019a) in different tectonic regimes. Subspace detectors, which are based on orthogonal basis waveforms, have been used for identifying earthquakes associated to aftershock sequences and low-frequency tremors (Maceira et al. 2010; Harris \& Dodge 2011).

Despite the fact that energy-based phase detectors do not require strong prior waveform knowledge, an increase in their ability to detect small onsets also implies higher false positive rates. Correlation detectors can achieve very low false positive rates, but they can solely detect similar signals to the ones already present in the selected template waveforms (Harris 1991). Subspace detectors can further extend the range of detected signals, depending on which detection threshold and subspace dimension parameters are used (see e.g. Harris \& Paik 2006). However, both correlation and subspace detectors are not easy to implement efficiently and hard to scale to very large data sets since they require a high computational cost.

All the above described methods exploit a priori assumed attributes of the signals such as energy or waveform similarity. Instead, deep learning methods learn signal characteristics directly from input data by applying non-linear transformations sequentially. In the supervised version of deep learning, the goal is to reduce the calculated error (objective function) between the network predictions and the known labels that the network is fed with (LeCun et al. 2015; Goodfellow et al. 2016). This is achieved by adjusting the connection weights between units in each layer after backpropagating the gradients of the objective function, computed over the 


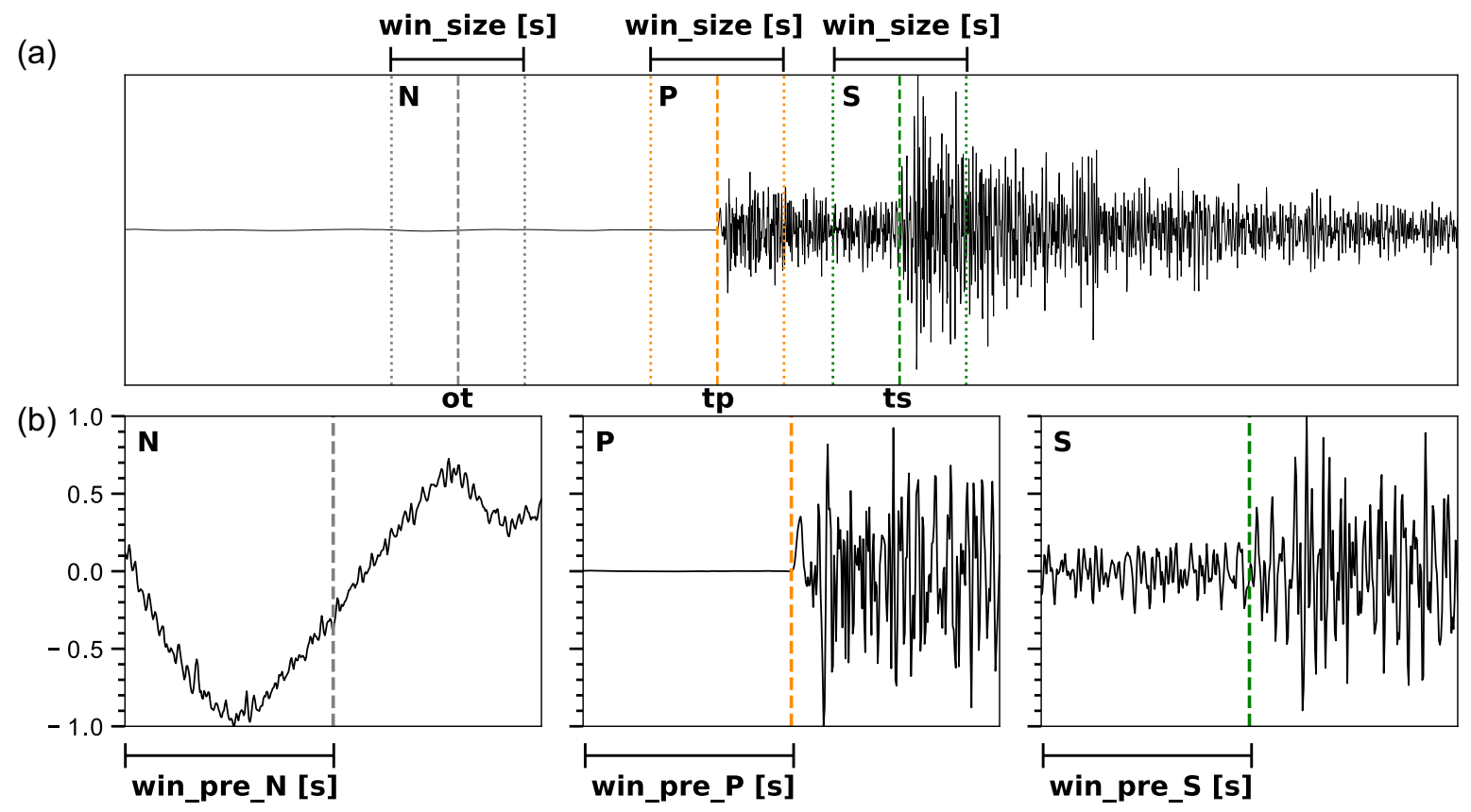

Figure 2. Seismic window extraction. (a) Example of an event waveform, outlining how the seismic window of three classes (N, P, S) are extracted for the optimized training of the phase detection model. N-, P- and S-class windows are extracted, respectively, in the vicinity of the event origin time (ot), and the true P- $(t p)$ and S- $(t s)$ onset times found by analysts. The hyperparameter win_size [s] defines the length of the extracted windows, which is equal for the three classes. (b) Zoom into the three-class windows. The starting time of the window before $o t, t p$ and $t s$ is defined by the hyperparameters $f r a c_{-} p r e \_N$, frac_pre_P and $\mathrm{rrac}_{-} \mathrm{pre}_{-} \mathrm{S}$ for classes $\mathrm{N}, \mathrm{P}$ and $\mathrm{S}$, respectively. Here win_pre_C $=$ win_size $\times$ frac_pre_C, where $C=N, P$ or $S$.

units in the last layer, to the input layer of the network. The network training consists of repeating the whole process for a fixed number of times (epochs) or until there is no further decrease in error (early stopping).

Convolutional neural networks (CNNs) are a class of deep neural networks specialized for processing unstructured data (Goodfellow et al. 2016). CNNs stand out due to the fact that they are computationally more efficient, easier to train, and have proven to effectively generalize learned features in many supervised tasks, ranging from image and document recognition (LeCun et al. 1990, 1998; Simard et al. 2003; Krizhevsky et al. 2012; Taigman et al. 2014) to seismic waves simulation (Moseley et al. 2018), or volcanic ash particles classification (Shoji et al. 2018).

Originally inspired by the properties observed in the primary visual cortex (PVC) of the mammalian brains (Hubel \& Wiesel 1959, 1962), modern CNN architectures are usually formed by several stages of consecutive operations of convolution, non-linear transformation, pooling, and regularization. In the first stage, a filter (kernel) performs local weighted sums (convolutions) through the input data. This linearly activates or detects local features, emulating the behavior observed in the so-called simple cells of the PVC. The resulting feature maps are transformed by applying a non-linear function. A further function then summarizes its statistics in a local scale (pooling). Usually the maximum (max pooling) within a subregion is computed in this second function, inspired by the function of PVC complex cells (LeCun et al. 2015; Goodfellow et al. 2016). Convolution is highly efficient and effective because it takes advantage of the local connectivity and invariance to location exhibited by meaningful learnable features in natural signals. First, it detects meaningful locally connected features by using kernels smaller than the input, which allows units in deeper layers to preserve information from the input layer. Secondly, it applies the same filters all over the input, thus permitting the learning of only one common set of parameters instead of many, as well as ensuring that the same learned representations can be found at different locations in the data. In addition to convolution, pooling in neighboring units in a layer can reduce the dimensions of the learned representation and makes it invariant to small perturbations in the input (LeCun et al. 2015; Goodfellow et al. 2016). Furthermore, regularization techniques help the learned features to better generalize to new unseen data, preventing model overfitting. One of the more effective and most frequently applied examples of regularization is Dropout (Srivastava et al. 2014), where noise is introduced in the output features of a layer aiming at inhibiting the network from learning non-meaningful patterns.

Traditional deep neural networks, including CNNs, are limited by having no memory, hence they cannot extract meaningful contextual information from sequentially structured data. Recurrent neural networks (RNNs; Rumelhart et al. 1986) overcome this constraint by simplistically mimicking human beings' mechanism for processing external information. They achieve this by implementing an internal loop that iterates over a sequence of data, element by element, while keeping an internal memory state of data they have already processed. The development of the long-short term memory RNNs (LSTMs) was one of the major breakthroughs in RNN architecture, since it solved the so-called vanishing gradient problem, whereby simple RNNs proved to be incapable of preserving longterm dependent information (Hochreiter \& Schmidhuber 1997). By carrying information to later points in the sequence, LSTMs are capable of retaining patterns, and therefore learning, from very long sequences. LSTM models make use of internal dropout and recurrent dropout that help prevent overfitting, so improving model performance. Another significant advancement was the invention of Bidirectional RNNs (BRNNs; Schuster \& Paliwal 1997), which 
Table 1. Hyperparameter search space optimized during the training of phase detection models. The second column lists the hyperparameter values that could be sampled during the optimization. The third column shows the best-performing value found after 1000 trials. The hyperparameter pre $m o d e$ defines the type of filter applied to the seismic data before entering the network as follows: pre mode $=1,2,3$ correspond to no filter applied, bandpass filter $(2-10 \mathrm{~Hz})$, and high-pass filter $(>0.2 \mathrm{~Hz})$, respectively. Hyperparameters

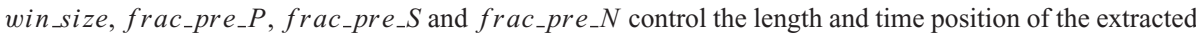
window samples used for the training, as described in the main text and Fig. 2. The range tested for the number of filters shown here relates only to the first convolutional layer. Another hyperparameter, not shown here, allows the number of filters in subsequent convolutional layers to be equal to or twice the value in the current layer.

\begin{tabular}{lll}
\hline Hyperparameter & Range tested & Best-performing value \\
\hline
\end{tabular}

Convolutional layers

Number of layers Number of filters

Kernel size

Activation function

Dropout

Dense layers

Number of layers

Number of units

Activation function

Dropout

Model training

Optimizer

Learning rate

Batch size

Data pre-processing

pre_mode
win_size $[s]$
frac_pre_P
frac_pre_S
frac_pre_N

$[1,2,3,4,5]$

$[2,4, \ldots, 30,32]$

$[3,5,7,9,11,13,15,17,19,21]$

[ReLU, sigmoid]

$[0.2,0.25,0.30,0.35,0.4,0.45,0.5]$

\author{
5 \\ $12,24,48,96,192$ \\ $17,11,5,9,17$ \\ ReLU, ReLU, ReLU, ReLU, sigmoid \\ $0.25,0.25,0.3,0.4,0.25$
}

make use of two recurrent layers to process sequences in both forward and backward directions. BRNNs can make learning more effective in sequences where both the past and future contexts can provide valuable insights.

In seismology, CNN models have been recently applied for detection (Ross et al. 2018a, b; Dokht et al. 2019; Woollam et al. 2019), association (McBrearty et al. 2019) and picking (Pardo et al. 2019; Zhu \& Beroza 2018) of $P$ - and $S$-wave arrivals, for earthquake localization (Kriegerowski et al. 2019; Perol et al. 2018; Mousavi \& Beroza 2020b; Zhang et al. 2020), event discrimination (Mousavi et al. 2019b) and magnitude estimation (Mousavi \& Beroza 2020a). RNN-based networks have been used for $P$-wave identification (Zhu et al. 2020), seismic phase association (Ross et al. 2019b) and for predicting approximate earthquake times and locations (Panakkat \& Adeli 2009). Model architectures based on combinations of $\mathrm{CNN}$ and RNN networks have been implemented for earthquake detection (Mousavi et al. 2019c) and combined event detection and phase picking (Zhou et al. 2019; Zheng et al. 2020) tasks. Recently, novel models which apply attention mechanism on a CNN+LSTM-based network architecture (Mousavi et al. 2020c) and utilize a capsule neural network (Saad \& Chen 2020) have also been used for combined event detection and phase picking.
This work adds to these previous studies, and introduces DeepPhasePick, an automatized two-stage method for detecting and picking seismic $P$ and $S$ phases from local earthquakes, entirely based on highly optimized deep neural networks. The first stage in DeepPhasePick consists of an adaptive CNN architecture trained for detecting the phases. Here the type of data pre-processing, as well as the length and position of the seismic phase windows used to train the network, were included among the optimizable hyperparameters. Phase picking is conducted in the second stage by applying two additional adaptive Bidirectional LSTM (BLSTM) networks, which were trained specifically to predict $P$ - and $S$-phase time onsets. The onsets, and their uncertainties, are determined on time windows defined based on the predicted probabilities of the $P$ and $S$ phases in the detection stage.

Here we demonstrate how the optimized CNN network in the first stage of DeepPhasePick, trained on a rather small data set of labelled phases in comparison to previous studies, is able to detect with high accuracy $P$ and $S$ phases from local earthquakes of different tectonic regimes. We also show how, by leveraging the information of the detected seismic phases, the optimized BLSTM models trained for picking are able to predict $P$ - and $S$-phase time onsets with analyst levels of precision, while also avoiding inherent human bias. 
(a)

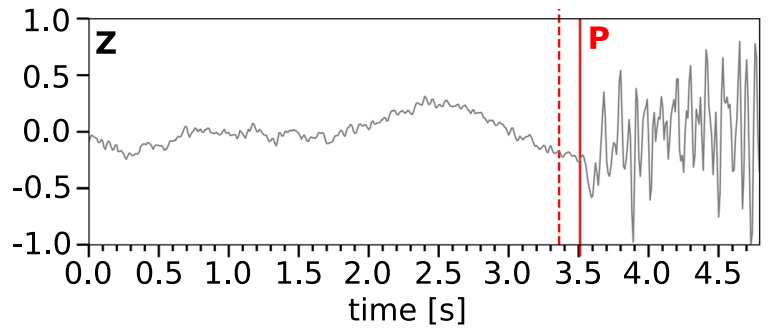

(c)

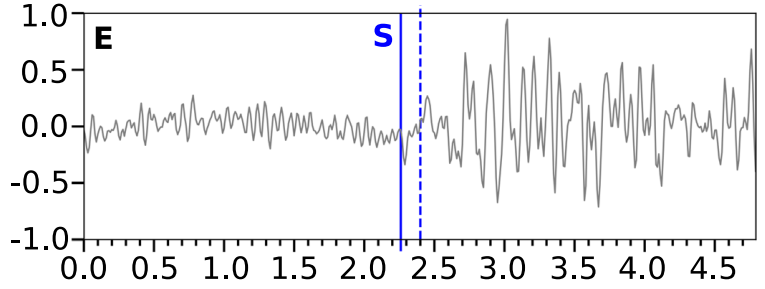

(e)

time [s]

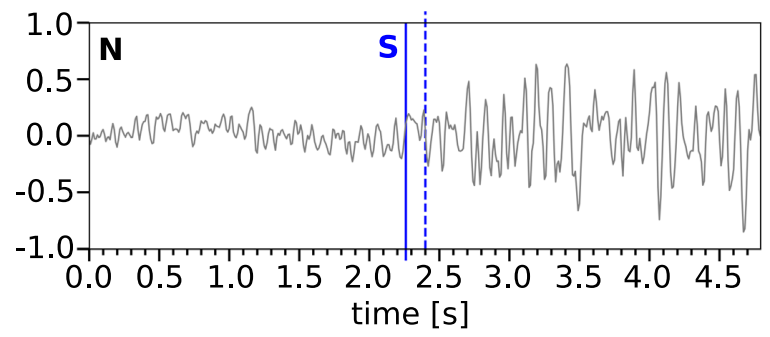

tons pred $=3.510$ tons err $=(-0.020,+0.010)$

(b) pick_class $=0 \quad \mathrm{pb}, \overline{\mathrm{pb}}$ _std $=(0.559,0.151)$

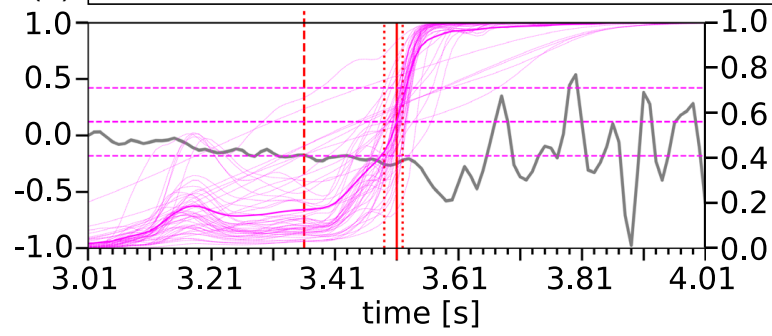

tons_pred $=2.260$ tons_err $=(-0.040,+0.120)$

(d) pick_class $=1 \quad \mathrm{pb}, \overline{\mathrm{pb}} \mathrm{std}=(0.516,0.126)$

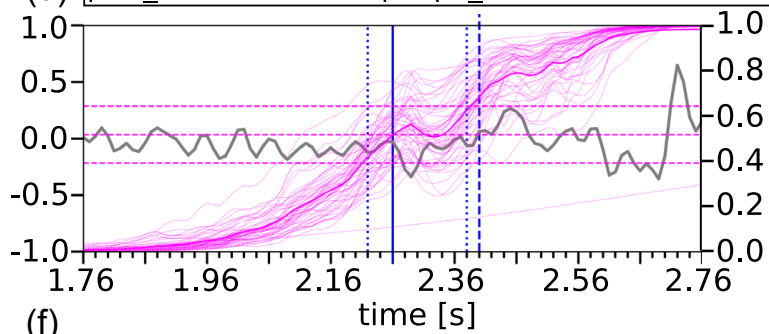

(f)

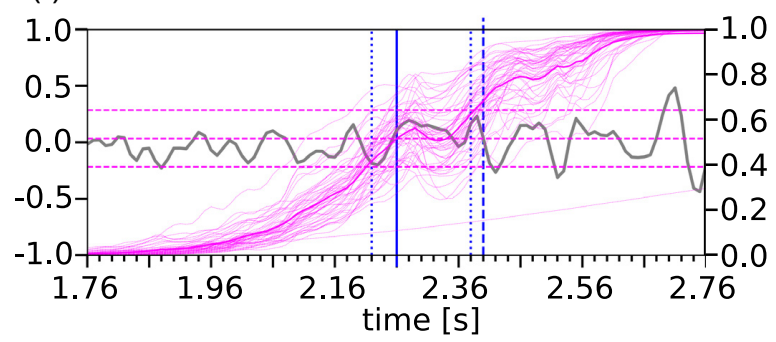

Figure 3. Estimation of time onset and associated uncertainty for predicted $P$ (a-b) and $S$ (c-f) phases. (a), (c), (e) Examples of predicted Z, E, N (Vertical, East, North) window samples, which correspond to the earthquake waveform shown in Fig. S1. Red and blue dashed lines represent the preliminary $P$ and $S$ onsets predicted in the phase detection stage, which were refined in the phase picking stage (red and blue solid lines). (b), (d), (f) Zoom in centred on the refined $P$ and $S$ time onsets (tons_pred [s]), estimated by applying MCD $(T=50)$ inference. Predicted class probabilities for each MCD prediction are plotted as magenta curves (right y-axis); bold curve represents the mean probability. Magenta horizontal dashed lines indicate the mean $(p b)$ and one standard deviation $\left(p b_{-} s t d\right)$ of the class probabilities at the onset time. As explained in the main text, from $p b$ and $p b_{-} s t d$, we can obtain tons_pred and its uncertainty (tons_err $[\mathrm{s}]$ ), outlined by red and blue vertical dotted lines before and after the refined $P$ - and $S$-phase onsets. Based on the weighting class scheme defined in Table 3 , $P$ and $S$ phases shown here correspond to picks of class 0 and 1 , respectively.

Table 2. Hyperparameter search space optimized during the training of $P$ - and $S$-phase picking models. The second column lists the hyperparameter values that could be sampled during the optimization. The third and fourth columns show the best-performing values found after running $50 P$ - and $S$-phase optimization trials.

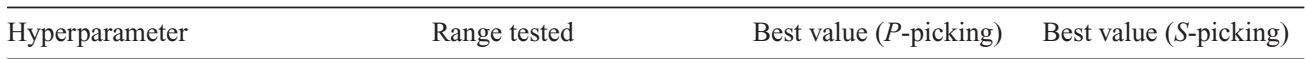

Recurrent BLSTM layers

Number of layers

Units

Dropout

Recurrent Dropout

Model training

Learning rate Batch size

[1e-05, 1e-04, 1e-03, 1e-02, 1e-01] $[50,60, \ldots, 190,200]$

\section{$[1,2]$}

$[10,20, \ldots, 190,200]$

$[0.2,0.25,0.30,0.35,0.4,0.45,0.5]$

$[0.2,0.25,0.30,0.35,0.4,0.45,0.5]$
100,160

$0.2,0.35$

2

$0.2,0.25$

$0.25,0.45$

$0.35,0.25$ 
Table 3. Weighting scheme for $P$ and $S$ predicted pick classes. Where terr $=\frac{1}{2}\left(\mid\right.$ tons_err ${ }^{-}|+|$tons_err $\left.^{+} \mid\right)$is the mean of the absolute time uncertainties calculated before and after the phase onset shown in Figs 3(b) and (d) insets.

\begin{tabular}{lc}
\hline Quality class & Time onset error $($ terr $[s])$ \\
\hline P0, S0 & terr $<0.05$ \\
P1, S1 & $0.05<$ terr $<0.1$ \\
P2, S2 & $0.1<$ terr $<0.2$ \\
P3, S3 & terr $>0.2$ \\
\hline
\end{tabular}

\section{DATA AND METHODS}

\subsection{Earthquake catalogue data sets}

In this study, we used two sets of manually picked event waveforms (Fig. 1). The first set (S1) consists of 1125 events from the time period between 1996-12-02T00:54:33.89 and 1997-1120T02:24:59.41 (Schurr et al. 2006). The second set (S2) contains 1196 events which occurred from 2007-06-14T01:18:52.24 to 2007-12-13T07:23:39.24 in the area of the 2007 M7.7 Tocopilla earthquake (Schurr et al. 2012). S2 contains mostly plate interface events, whereas S1 contains mostly intermediate-depth intraplate events.

From these earthquake catalogues, we extracted three-component seismograms which we subdivided into three window classes: $25647 P$-phase, 25647 Noise $(\mathrm{N})$ and $14397 S$-phase windows. $16234 \mathrm{P}, 16234 \mathrm{~N}$ and $8061 \mathrm{~S}$ of these samples were obtained from $\mathrm{S} 1$, and $9413 \mathrm{P}, 9413 \mathrm{~N}$ and $6336 \mathrm{~S}$ samples from S2. We used these seismic windows as input data to train adaptive neural networks in phase detection and phase picking tasks, as will be described in the next sections.

\subsection{Hyperparameter optimization of adaptive neural networks}

The architecture of a neural network is defined by its hyperparameters, such as the number of layers in the network, the training learning rate and the batch size used during training. The selection of the model hyperparameters is key when implementing a supervised deep learning task, since it may lead to a significant improved performance of the trained model, especially when the available data is limited.

Commonly used hyperparameter optimization approaches, which rely on grid or manual search, have been shown to be less efficient than an optimization based on random search (Bergstra \& Bengio 2012). However, all the above mentioned methods select the subsequently sampled values without an informed criterion. This makes the optimization less effective, since the sampling of hyperparameter values which do not lead to improved performance tends to require a significant amount of time. In contrast, Bayesian optimization selects the next sampled hyperparameters based on previous evaluations. This has proven more efficient in terms of balancing exploration-exploitation of the search space, time consumption and model performance results, compared to random search (Bergstra et al. 2013a; Hinz et al. 2018).

In Bayesian optimization, an objective function is minimized by mapping past evaluations of the hyperparameters to a surrogate probabilistic model of the objective function, which is then more simply optimized instead. In this work, we used a Bayesian approach that optimizes a surrogate model defined by the Tree Parzen Estimator (TPE) algorithm, as implemented in the Python library
Hyperopt (Bergstra et al. 2013b). Basically, TPE algorithm applies the Bayes rules to create two different probability distributions for the hyperparameters, depending on the score reached by the objective function. The next set of sampled hyperparameters is then selected based on the expected improvement in the objective function (Bergstra et al. 2011).

We implemented two types of objective functions, which are minimized in order to optimize the training of different adaptive neural network architectures designed to solve two tasks: phase detection and phase picking. These architectures will be described in detail in the next sections. The hyperparameter optimization process was performed using one GPU NVIDIA GeForce RTX 2080. Multiple iterations (trials) were run for each task, with a different hyperparameter configuration being attempted in each trial, until a best-performing trained model, that is a model with minimum error, was found. The adaptive neural networks were built in Python using the machine learning framework Keras (Chollet 2015) with Tensorflow as backend (Abadi et al. 2015).

\subsection{Phase detection as a supervised multiclass classification task}

We implemented the phase detection stage as a supervised multiclass classification task, based on an adaptive neural network formed by two blocks of deep layers. The first block is made up of between one and five convolutional layers, which extract abstract representations (features) from three-component seismograms input (samples) and help reduce their dimensionality. The output of the convolutional layers is then flattened before entering the second block, formed by between one and four dense (fully connected) layers. All the units in a fully connected layer are connected to all the units in the preceding layer. This allows them to better learn correlated features throughout the input data, rather than only the locally connected features learned by convolutional layers. Hence, dense layers are well suited for the final classification stage in the adaptive network. Since the network is adaptive, the number of layers in each block, as well as specific layer variables such as the number of convolutional filters or dense units, are adjusted during the hyperparameter optimization.

We chose to use depthwise separable 1-D convolutional layers for the feature extraction in the first block. Depthwise separable convolutional layers implement a 2-step convolution process, first performing independent convolutions on each channel of the input data and then combining individual channel outputs through a pointwise $(1 \times 1)$ convolution (Chollet 2017). This type of convolution is appropriate for learning patterns from multicomponent seismic data, such as 1-D amplitude time-series from seismic waveforms, since different features can be extracted from the three input channels (the three components of each seismogram) independently. This may help the network extract specific patterns from, for example the two horizontal components, in order to better identify $S$ phases. Furthermore, compared to standard convolutional layers, depthwise separable layers have the additional advantage of helping reduce model overfitting, since they have fewer weights to adjust, and therefore require fewer calculations. This further reduces the overall computational cost, since less calculation time is required to complete the multiple iterations over different network architectures involved in the hyperparameter optimization.

Outputs from each of the convolutional and dense layers in the network, except from the output of the final dense layer, are passed through a layer that applies a non-linear activation function that 

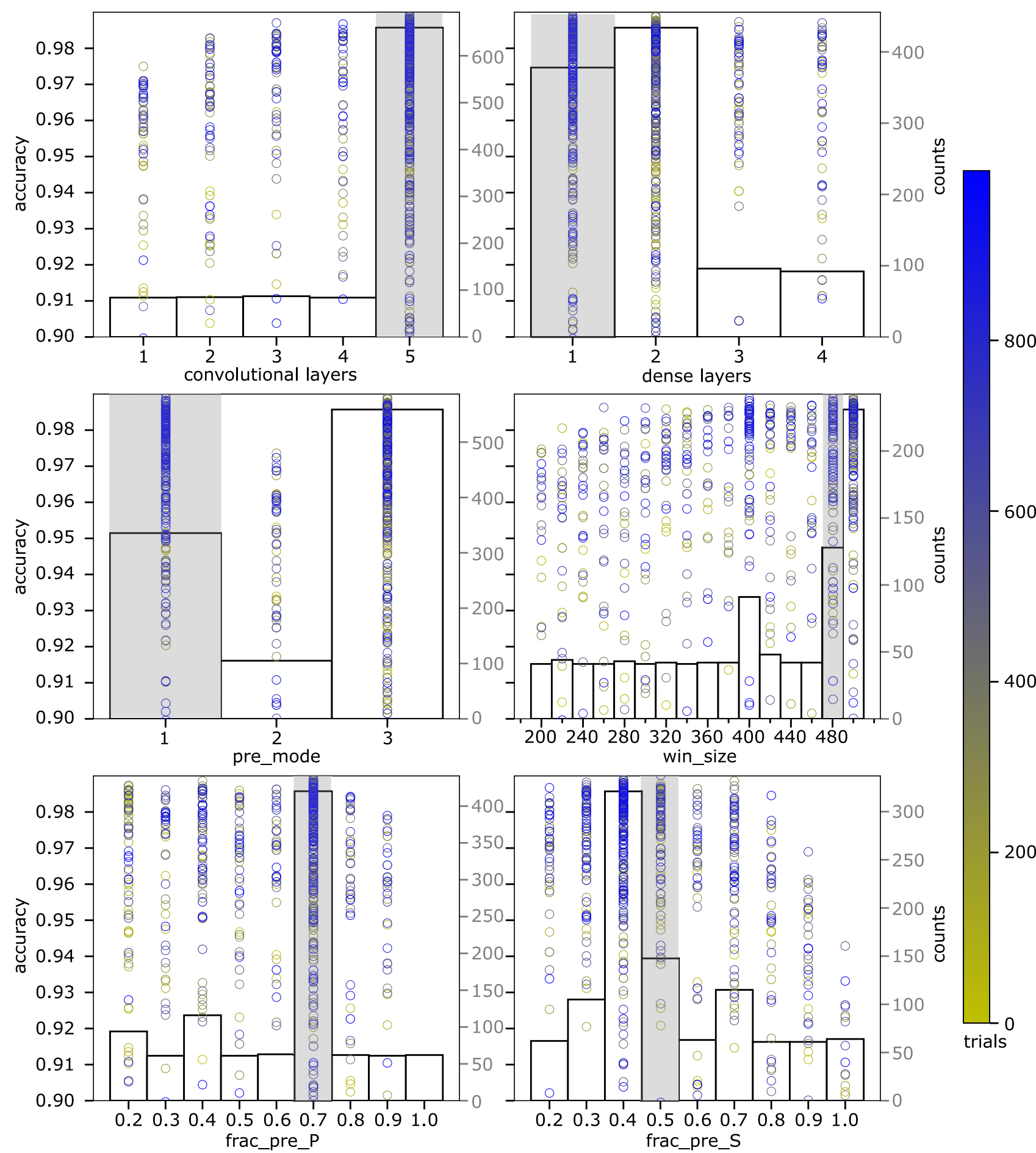

Figure 4. Distribution over the 1000 optimization trials of the six main hyperparameters used in the phase detection model training. Subplots show sampled hyperparameter values coloured by trial number and plotted versus the model accuracy reached at that trial (left $y$-axis). The distribution of the hyperparameter values sampled over the trials is presented as a histogram (right $y$-axis). A grey shaded bin in the histogram represents the best-performing hyperparameter value, that is, the value with which the model reached the highest accuracy.

can be either a rectified linear unit (ReLU; Nair \& Hinton 2010) or a sigmoid, according to the hyperparameter selection. Additional Batch Normalization (Ioffe \& Szegedy 2015) and Dropout (Srivastava et al. 2014) layers are stacked to the activation outputs in both blocks. In the convolutional block, a further 1-D Max Pooling layer is applied between each Batch Normalization and Dropout layer. The output of the final dense layer is passed instead through a softmax activation function. This outputs a vector of three probabilities, adding up to 1.0, each expressing the likelihood of a sample belonging to one of the three possible classes tested: P, S or N. The greatest among these probabilities determines the predicted class of the three-component record. 

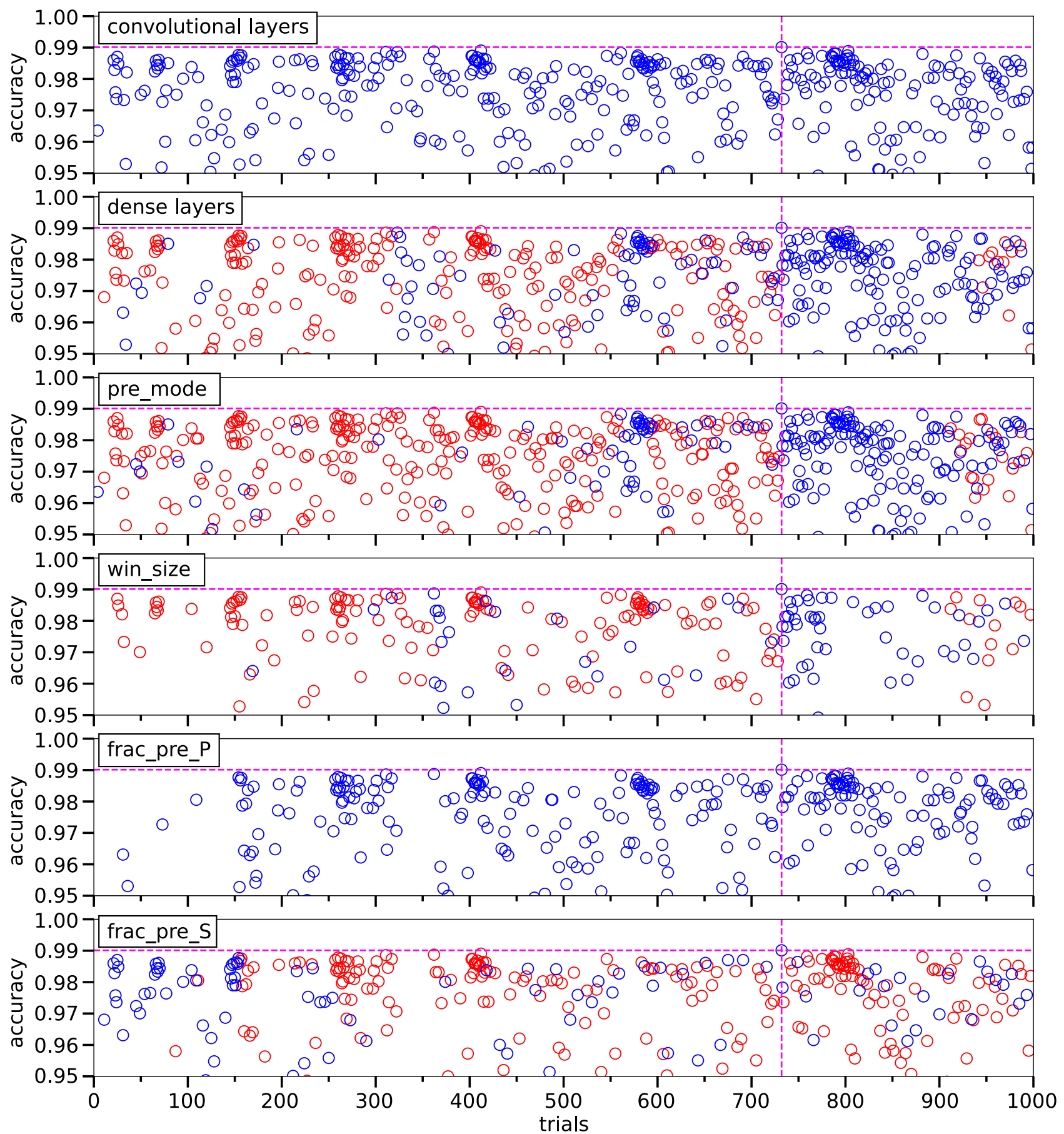

Figure 5. Each subplot depict in detail the distribution of the best-performing hyperparameter values (grey shaded bins in histograms of Fig. 4; blue circles) that led to higher model accuracies $(>0.95)$ over the trials. Values plotted are the accuracies on the validation set, reached at the end of each trial training. For the hyperparameters in which the best-performing value does not coincide with the most frequently sampled value, the latter are plotted as well (red circles). For reference, magenta horizontal and vertical dashed lines mark the highest model accuracy $(0.990)$ reached during the optimization and the trial (733) at which it was achieved, respectively.

Prior to initializing the optimized training, we randomly extracted an independent test set consisting of $1440 \mathrm{P}, \mathrm{S}$ and $\mathrm{N}$ threecomponent time-series windows, made up of 888 records from $\mathrm{S} 1$ and 552 records from $\mathrm{S} 2$ data sets for each class, so as to keep the proportion of samples present in each data set. In order to reduce the possibility of biased phase classification due to an imbalanced class distribution in our data set ( 39 per cent $\mathrm{P}, 39$ per cent N, 22 per cent $\mathrm{S})$, the remaining data was balanced out at each optimization trial by randomly discarding the surplus samples of the over-represented $\mathrm{P}$ and $\mathrm{N}$ classes. Then, the balanced data was shuffled and assigned to the training and validation sets before carrying out the model training. The resulting training, validation and test sets added up to 75 per cent (32 393), 15 per cent (6478) and 10 per cent (4320) of the total balanced data samples, respectively. During the training, features of the waveforms were learned from the training set and the weights in the network layers were adjusted based on this. The 

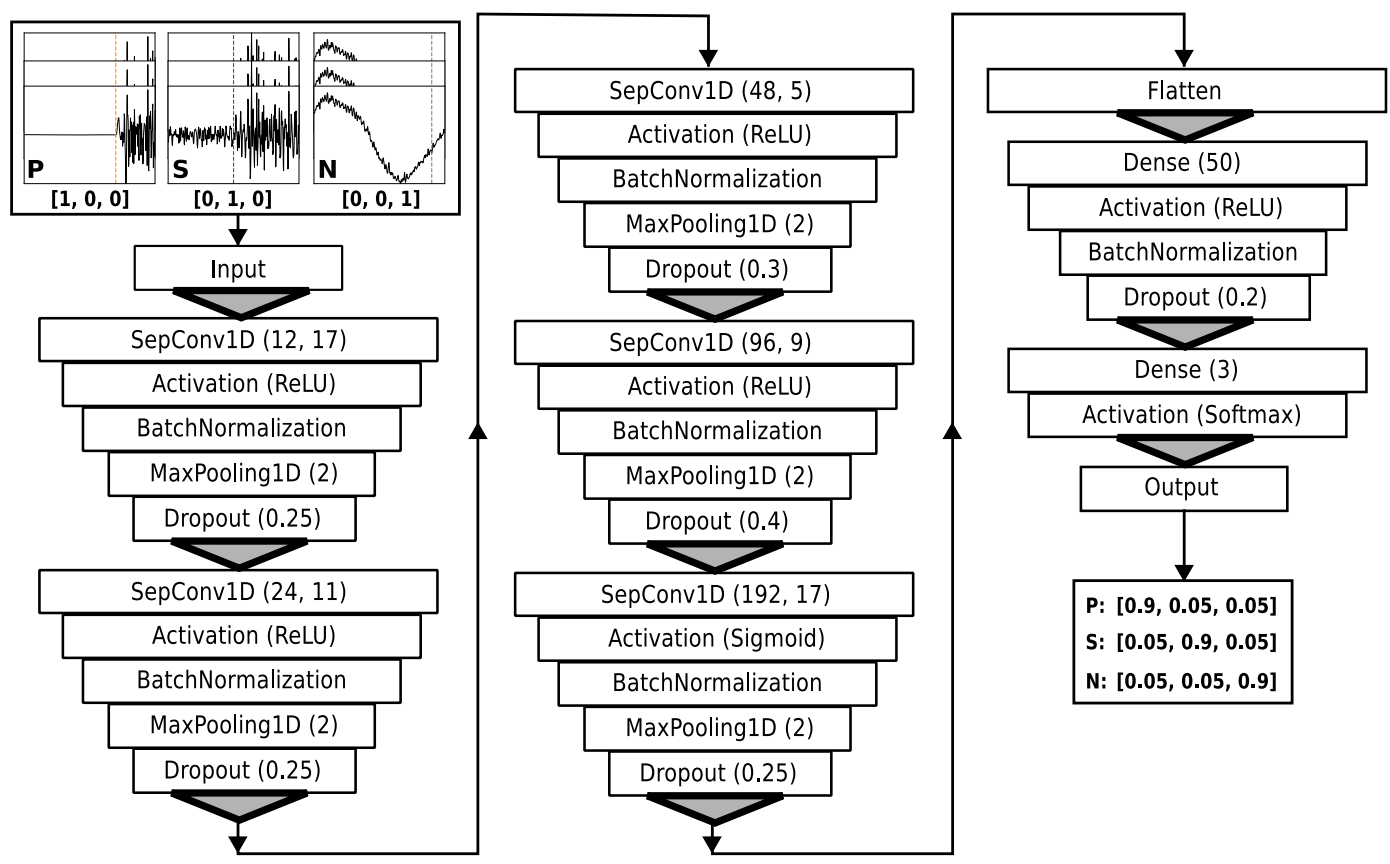

Figure 6. Optimized model architecture trained for phase detection task. The network receives as input class vectors representing the respective accompanying three-component seismogram samples and outputs three-probability vectors indicating the predicted classes (P, S or N). The optimized hyperparameters in each block of convolutional and dense layers are shown here and also presented in Table 1.
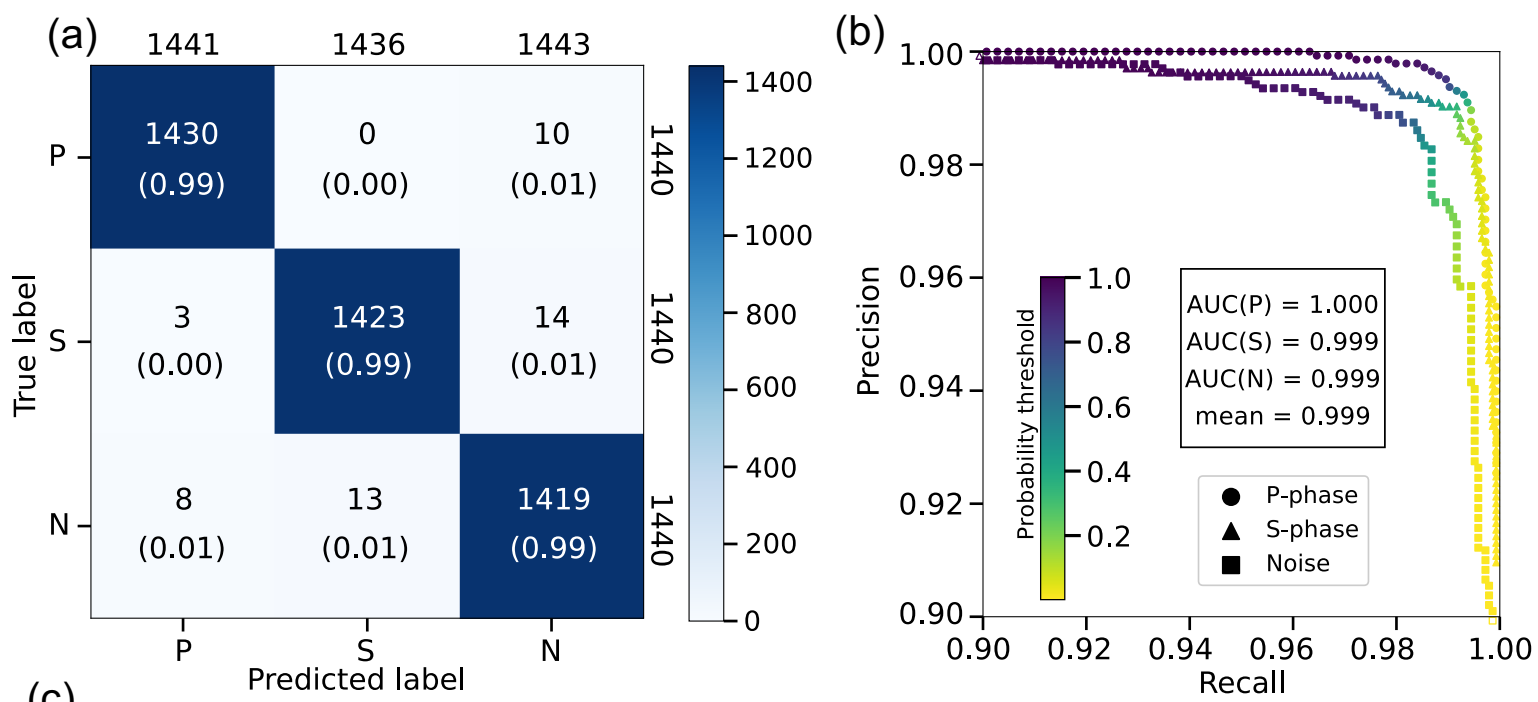

(c)
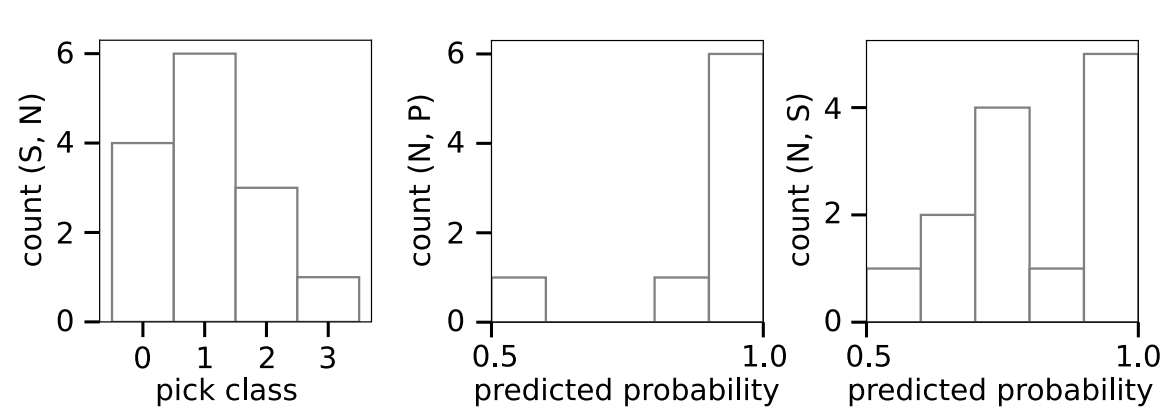

Figure 7. (a and b) Results from evaluation of the best phase detection model on the independent test set of 4320 samples, represented in a confusion matrix (a) and a precision-recall curve (b). In (b), AUC represents the area under the precision-recall curve for each class. Precision, Recall and F1-score at two probability thresholds are reported in Table S1. (c) disaggregated histograms of incorrectly predicted $P$ or $S$ phases as noise and presumably misclassified noise samples as $P$ or $S$ phases. (P, N) means $P$-phase samples predicted as Noise, and similarly for other cells in the confusion matrix. 
(a)

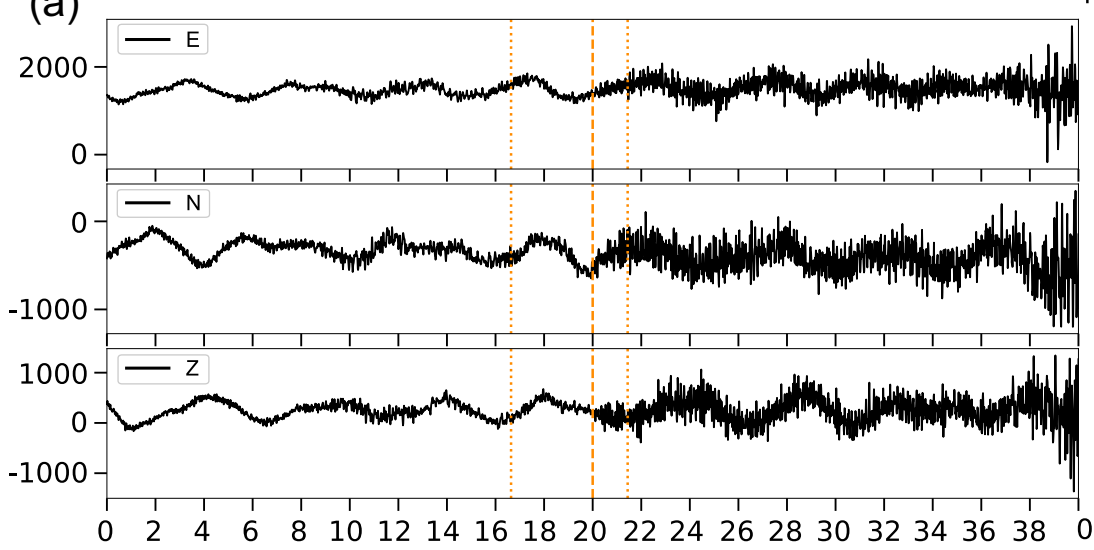

(b)

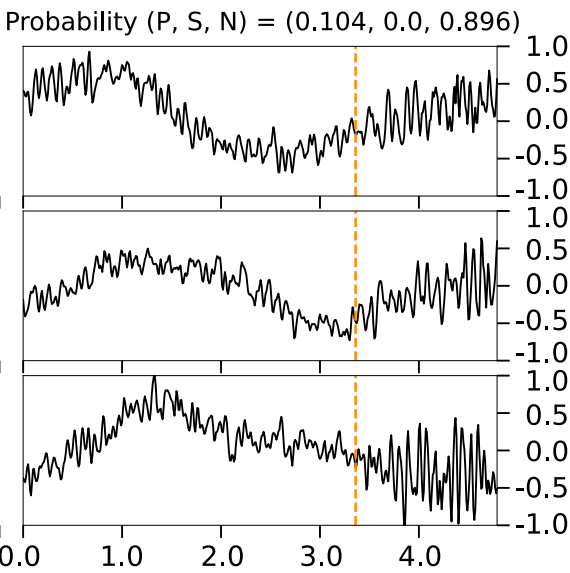

Probability $(P, S, N)=(0.006,0.06,0.934)$

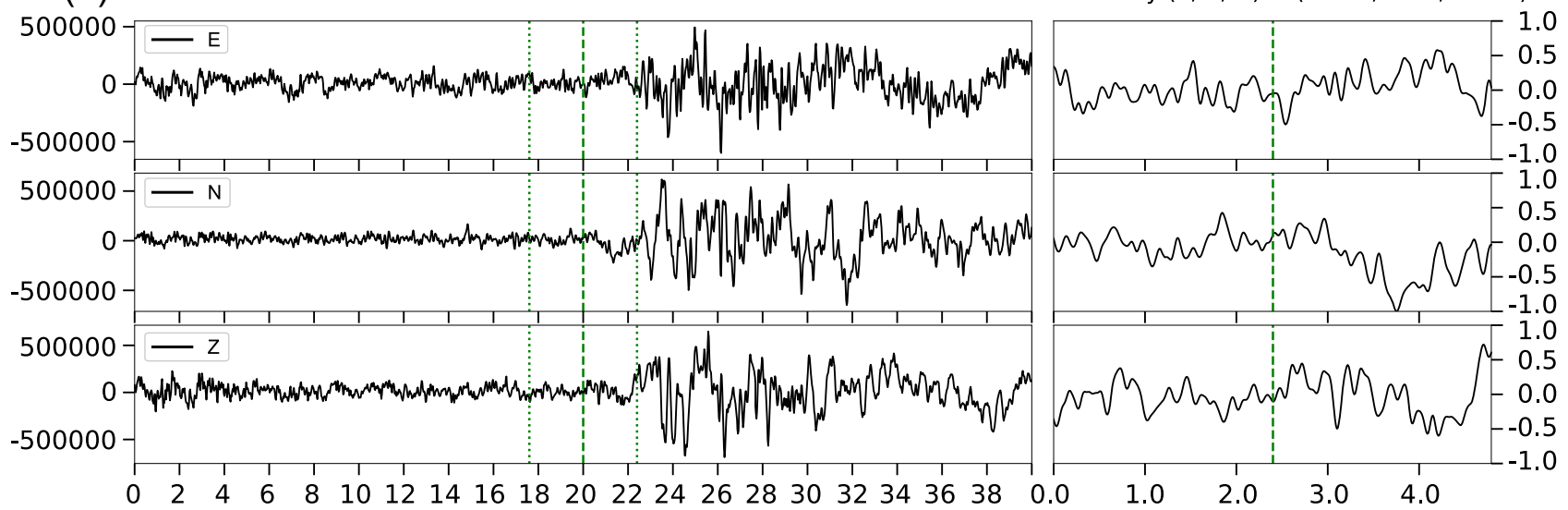

Figure 8. (a) Example of $P$-phase sample in the test set of 4320 samples, mispredicted as noise (N). (b) Example of S-phase sample in this test set, mispredicted as noise. The left subplots in (a) and (b) show the three-component seismic waveform from which the samples were extracted. The extracted samples, as received by the network, are shown in the right subplots. Examples shown here correspond to $P$-phase and $S$-phase samples with analyst's pick weights 2 (50 per cent confidence) and 1 ( 75 per cent confidence), respectively.

updated model was then used to predict the sample classes in the validation set, and adjust the model hyperparameters accordingly. The final performance of the best trained model was evaluated using the test set. We assigned to each sample in the training, validation and test sets, binary vectors representing the sample class as follows: $[1,0,0]$ for $\mathrm{P}$ class $[0,1,0]$ for $\mathrm{S}$ class, and $[0,0,1]$ for $\mathrm{N}$ class. These vectors were used as the known labels which were compared with the vector of class probabilities predicted by the model.

During each optimization trial, seismic data was linearly detrended, resampled at $100 \mathrm{~Hz}$ and filtered. The type of filter applied to the data is defined by the currently sampled value of the hyperparameter pre_mode, and can be band-pass $(2-10 \mathrm{~Hz})$, high-pass $(>0.2 \mathrm{~Hz}$ ), or no filter. Next, the sample windows were extracted as defined by the sampled win_size, frac pre $_{-} N$, frac_pre_P and frac_pre_S hyperparameter values. The hyperparameter win_size defines the length of the extracted windows (2-5 [s]), which we imposed to be equal for the three classes. This makes data manageable by the network during the training. The three additional hyperparameters $\left(f r a c_{-} p r e_{-} N\right.$, frac $c_{-} r_{e_{-}} P$ and $f r a c_{-} p r e_{-} S$ ) vary between 0.2 and 1.0 and define the fraction of the extracted windows that is placed before the event origin time, the true P-phase onset and the true S-phase onset for classes N, P and S, respectively (see Fig. 2). Then the extracted sample windows were normalized by the maximum amplitude across the three waveform components and subdivided in the training, validation and test sets, as explained above.
The training and validation sets were then used to train the model configuration defined by the rest of the hyperparameters sampled in the trial.

The total space of hyperparameters searched during the optimization is presented in Table 1. In order to search for the bestperforming network trained in detecting seismic phases, we ran 1000 hyperparameter optimization trials $(\sim 9 \mathrm{~min}$ per trial). In each trial we used the validation accuracy as the optimizable metric and we trained the model for up to 60 epochs using the categorical cross-entropy loss function. To speed up the training process, we additionally applied an early stopping callback that stopped the training if the validation accuracy did not increase in 6 epochs.

\subsection{Phase picking as a supervised sequence binary classification task}

As mentioned previously, phase picking in DeepPhasePick uses two optimized adaptive network architectures, each formed by one or two BLSTM layers, which we implemented as a supervised sequence binary classification task.

We trained a first model for picking $P$ phases, using as input the amplitude time-series taken from vertical-component seismograms. A second model was trained for picking $S$ phases, based on the amplitude time-series of the two horizontal-component records. We trained each model to learn patterns from seismic data that account 
(a)

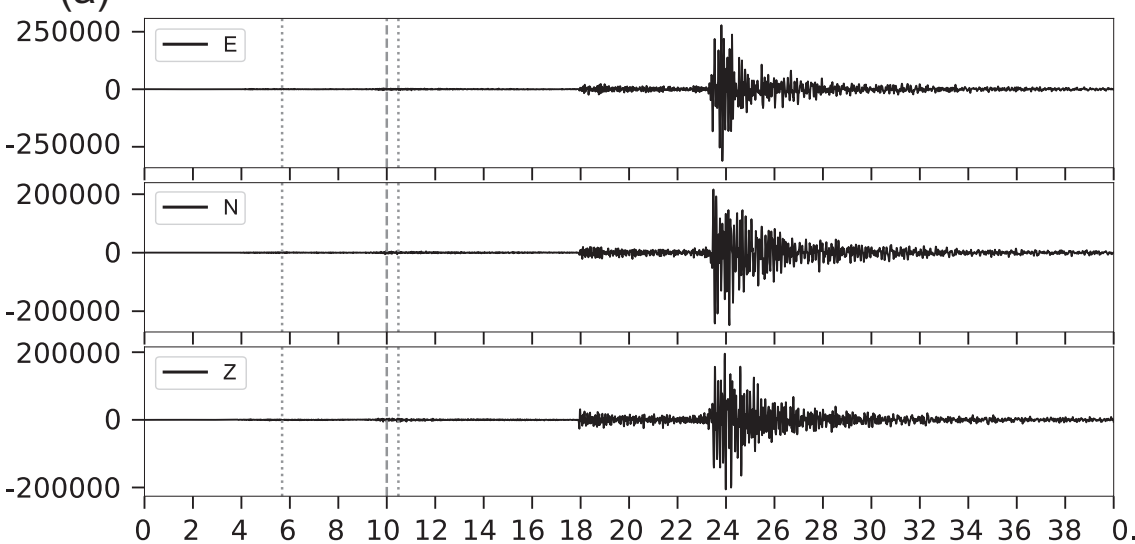

Probability $(P, S, N)=(0.904,0.004,0.092)$

(b)
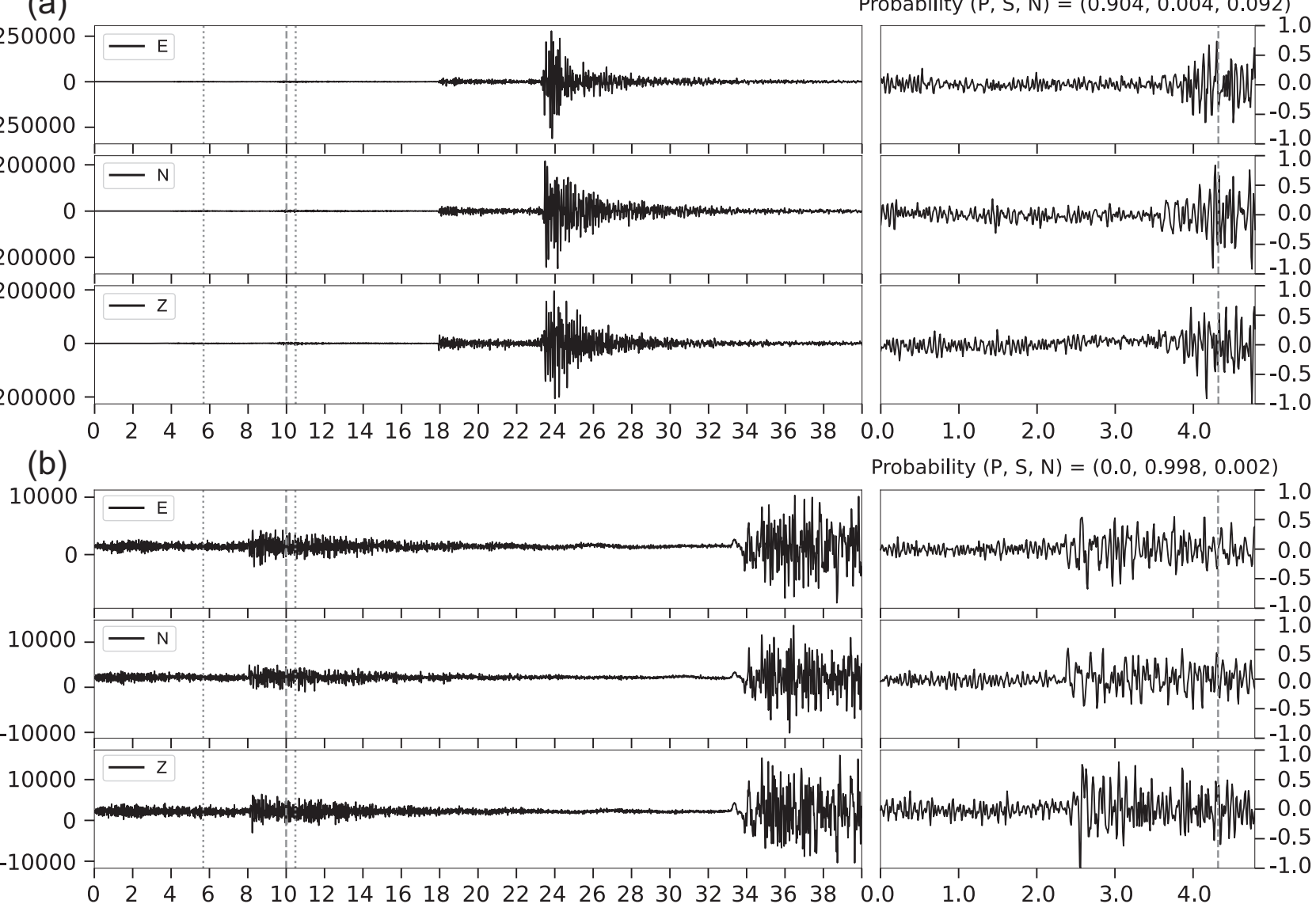

Probability $(P, S, N)=(0.0,0.998,0.002)$

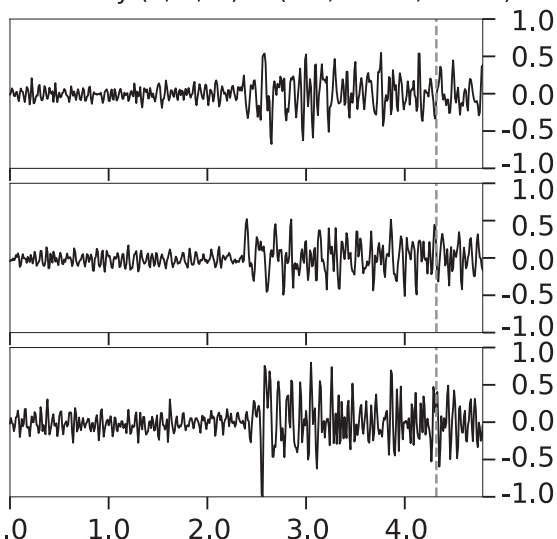

Figure 9. (a) Example of noise $(\mathrm{N})$ sample in the test set of 4320 samples, seemingly mispredicted as a $P$-phase. (b) Example of noise sample in this test set, seemingly mispredicted as a $S$-phase. Subplots are plotted the same way as in Fig. 8. The apparent misclassifications shown here are examples of non-picked phases, which were however detected by the network.

for the transition from noise to signal in both $P$ and $S$ phases from local earthquakes, therefore allowing identification of $P$ and $S$ time onsets. BLSTM-based networks are suitable for such task, since they are able to retain and learn sequential characteristics while processing input seismic time-series in both chronological and antichronological order, hence learning dependencies between phase onsets and neighboring seismic patterns. A final dense layer in both picking models applies a sigmoid activation function, which outputs the probability of the time steps in the time-series corresponding to either noise or seismic signal.

Before carrying out the model training, 10 per cent of the window samples were randomly extracted as an independent test set. As in the phase detection stage, the test set includes a proportional amount of records from S1 and S2 data sets. The remaining samples were first shuffled and then subdivided into training (65 per cent) and validation ( 25 per cent) sets. Altogether, this corresponds to 16670 training, 6412 validation and 2565 (1625 from $\mathrm{S} 1$, and 940 from S2) test one-component samples for P-picking; and 9358 training, 3599 validation and 1440 (808 from S1 and 632 from S2) test two-component samples for S-picking. As in the phase detection stage, weights and hyperparameters of the models were adjusted based on the training set and validation set, respectively. The test set was then used to evaluate the performance of the best trained models. The window samples were defined based on the optimized hyperparameter values for the phase detection model, namely the window length (win_size) and its relative position with respect to the true onset (frac_pre_P and frac_pre_S). This allows the integration of phase detection and phase picking in a common two-stage workflow, with the aim of making the overall process more efficient, since both stages share already optimized information.

However, instead of using the above-described seismic windows, we trained and validated the picking models on versions of them, which were randomly shifted in time by a range between -0.5 and 0.5 [s] around the true $P$ and $S$ onsets. This allows the networks to better learn data patterns in the vicinity of the phase onsets, and so preventing the model from memorizing only fixed onset times. A similar approach has been applied by Ross et al. (2018a) by using an artificially augmented number of samples to train a CNN network as regressor for $P$-phase picking. In the test set, we kept both the original time windows and their shifted versions, in order to test the detection capacity of the trained models in both scenarios.

So as to perform the model training, we assigned to each input sample a binary vector of length equal to the sample window length. This vector is a binary representation of the ground truth, where all the 0 s correspond to time steps associated to noise, and all the 1s representing signal, so that the first 1 represents the time step of the true phase onset. Once trained, the models output a vector of the same length, which contains numbers between 0 and 1.0 corresponding to binary class probabilities: $<0.5$ for time steps predicted as noise and $>0.5$ for time steps predicted as signal. Thus, we can determine the predicted phase time onset in a given sample 
(a)

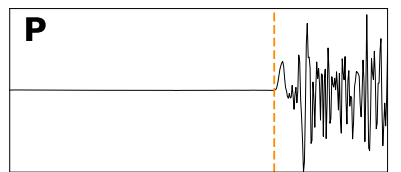

$[0,0, \ldots \ldots \ldots \ldots \ldots, 0,0,1, \ldots \ldots, 1]$

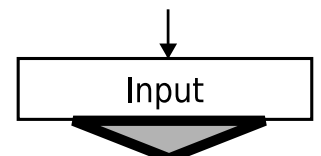

Bidirectional

$\operatorname{LSTM}(100,0.2,0.2)$

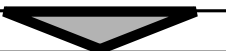

Bidirectional

$\operatorname{LSTM}(160,0.35,0.25)$
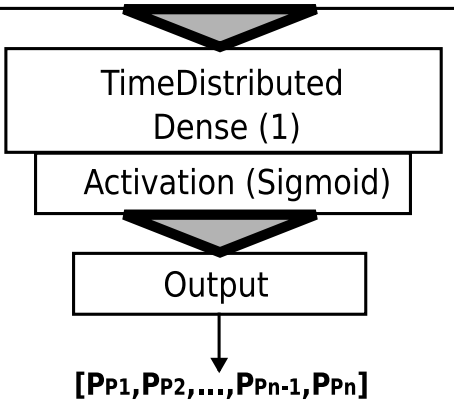

(b)

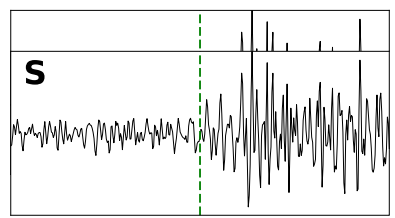

$[0,0, \ldots \ldots \ldots, 0,0,1,1, \ldots \ldots, 1,1]$

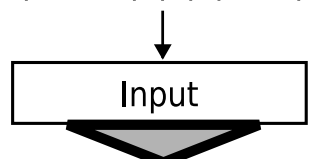

Bidirectional

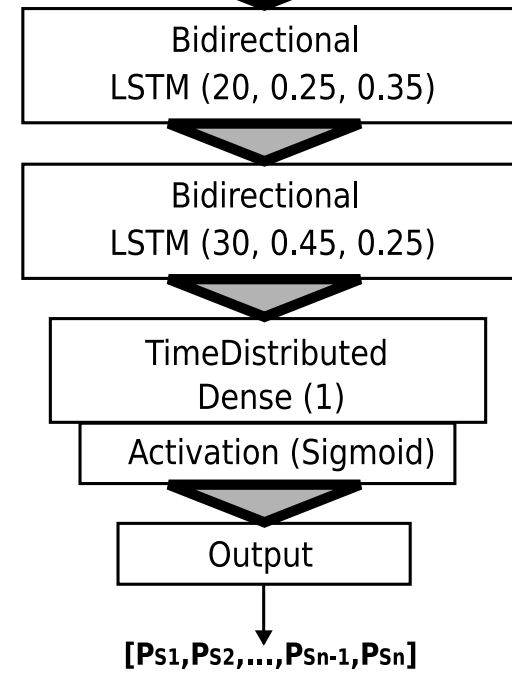

Figure 10. Optimized model architectures trained for $P$-phase (a) and $S$-phase (b) picking tasks. The networks receive as input binary vectors representing the noise (0) and signal (1) content in accompanying time-series taken from the vertical-component (a) and the two horizontal-component (b) seismograms of the samples used for training. The models output is the probability of the time steps in input samples corresponding to either noise or seismic signal. The optimized hyperparameters in each BLSTM layer are shown as (Units, Dropout, Recurrent dropout), which are also reported in Table 2 for both models.

as the time step where the output probabilities first rise above 0.5 , indicating a change from noise to signal.

We ran 50 hyperparameter optimization trials for the $P$ - $(\sim 130$ min per trial) and $S$-phase ( $\sim 50$ min per trial) picking tasks. Table 2 presents the hyperparameter space searched during the optimization. We optimized on the validation accuracy metric, using the binary cross-entropy loss function and the Adam stochastic optimization algorithm (Kingma \& Ba 2014) in the training. The training was carried out using the same number of epochs and similar early stopping as in the phase detection stage.

\subsection{From predicted phase probabilities to preliminary onsets}

By applying our best-performing models trained for both phase detection and phase picking on continuous seismic data, we were able to obtain the time onsets of $P$ and $S$ phases originating from local earthquakes. In this section we explain how we first obtained preliminary time onsets in the phase detection stage. The next section describes how we refined them in the phase picking stage to estimate the final phase onsets and their associated uncertainties.

First, we used the optimized phase detection model to predict the class probabilities of three-component moving windows. By iteratively shifting these windows along the continuous seismic waveforms, we obtained a discrete probability time-series of each class. The length of the moving window is given by the optimized hyperparameter winsize. We assigned the predicted probability at the position within the window defined by the optimized hyperparameters $f r a c_{-} p r e_{-} N$, $f r a c_{-} p r_{-} P_{-}$and $f r a c_{-} p r e_{-} S$ for the respective classes (see Table 1, and Figs 2 and S1). The time step between consecutive moving windows is a user-defined parameter that controls the resolution of the probability time-series.

Next, we declared a $P$-phase search window between the time where the P-class probability time-series $\left(p b_{-} P\right)$ rises above certain trigger threshold $p b_{\_} P_{-} t h 1$ and the time where $p b_{-} P$ decreases below a certain detrigger threshold $p b_{-} P_{-} t h 2$. We estimated a preliminary $P$-phase onset as the time of the maximum $p b_{-} P$ within the search window. For waveforms where a preliminary $P$ onset is found, we followed a similar approach to define a preliminary $S$-phase onset at the time of the maximum $S$-class probability time-series $\left(p b_{-} S\right)$, within an $S$-phase search window delimited by the time interval that satisfies the condition $p b_{-} S_{-} t h 1<p b_{-} S<p b_{-} S_{-} t h 2$. Cases where $p b_{-} P$ and $p b_{-} S$ overlap or are close enough in time at values above the trigger thresholds cannot be handled solely with the previous criteria. We included few additional conditions, based on the predicted probabilities, to deal with this and other particular scenarios. These conditions can be optionally activated by the user and depend on a few user-defined parameters (see Text S1).

\subsection{Estimation of final phase onset times and their uncertainties}

The preliminary $P$ and $S$ onsets were defined based on probabilities returned by a model trained for recognizing seismic phases, rather 

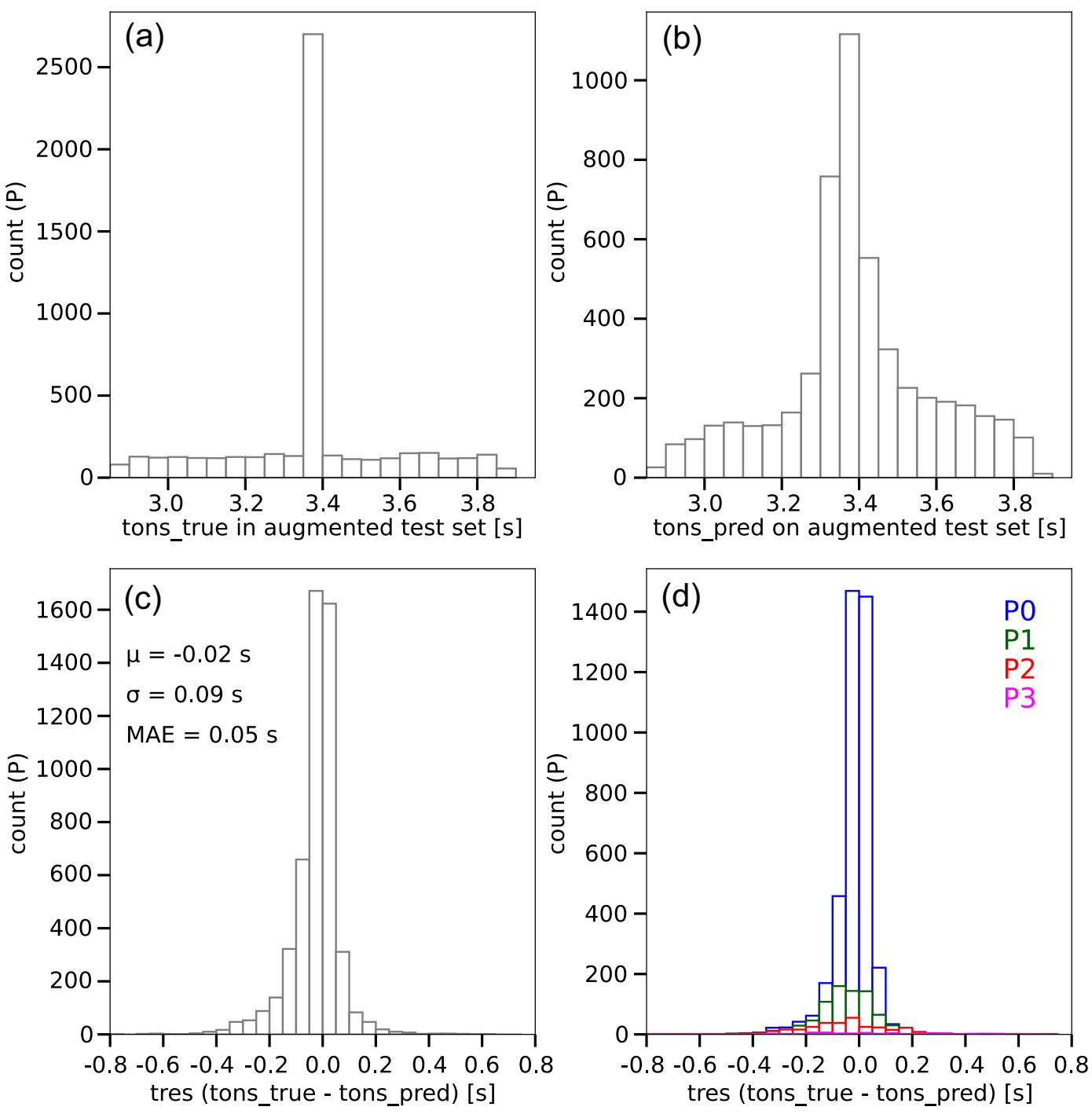

Figure 11. Evaluation of the optimized model trained for $P$-phase picking on the independent augmented test set of $2565 \times 2$ one-component waveform samples. (a) True time onsets (tons_true) from both the actual and shifted samples forming the augmented test set. (b) Predicted time onsets (tons_pred, defined as in Fig. 3) by the best model trained for $P$-phase picking. (c) Time residuals (tres) distribution, defined as the difference between the true and predicted time onsets. Reported mean $(\mu)$, standard deviation $(\sigma)$ and mean absolute error (MAE) are calculated on this residual distribution $(\mid$ tres $\mid \leq 0.8)$. Here we considered MAE $=\frac{1}{n} \sum_{i=1}^{n}\left|t_{i}-\hat{t}_{i}\right|$, where $t_{i}$ and $\hat{t}_{i}$ are the predicted and true onsets of $n$ samples, respectively. (d) Predicted pick quality classes, as defined by weighting scheme defined in Table 3.

than for actually picking them, and therefore need to be refined. To this end, we used 'picking windows' of length win_size, as defined by the optimized hyperparameters $\mathrm{frac}_{-} \mathrm{pre}_{-} \mathrm{P}$ and $\mathrm{frac} \mathrm{c}_{-} \mathrm{pre}_{-} S$ around the preliminary $P$ - and $S$-phase onsets. On these picking windows, we then applied the optimized models trained for $P$ - and $S$-picking to estimate the respective phase onsets as well as the associated uncertainties.

Uncertainties were determined by applying the stochastic regularization technique Monte Carlo Dropout (MCD). It has been shown that using dropout at testing time of a deep neural network can be interpreted as a Bayesian approximation of a Gaussian process, hence MCD can be used as an approximation of Bayesian variational inference (Gal \& Ghahramani 2016a, b). This means that a model posterior distribution for a given sample can be represented by multiple (T) predictions made by the network with dropout. Thus, the value and uncertainty of the predicted sample can be obtained as the mean and standard deviation of the $\mathrm{T}$ inferences.
In practice, we implemented MCD by performing T model predictions with dropout. In this case, since phase picking is treated as a sequence binary classification task, MCD generates a collection of predicted class probabilities of the time steps in an input timeseries. This collection is an approximation of the model posterior distribution for that input sample, which is described by the mean and standard deviation of the $\mathrm{T}$ class probabilities at each of the sample time steps.

Then, we estimated the predicted phase time onset (tpred) for the sample as the first time step in which the mean of the class probabilities rises above $0.5(\mathrm{pb})$, which indicates the predicted transition from noise to signal. The standard deviation of the class probabilities at tpred ( $\left.p b_{-} s t d\right)$ represents the variability of the predicted probability at the assumed time onset. We propose that a reasonable first order estimation for the time onset uncertainty can be determined as the interval of time steps delimited before and after tpred by the projection of $p b s t d$ on the mean class probability (see 

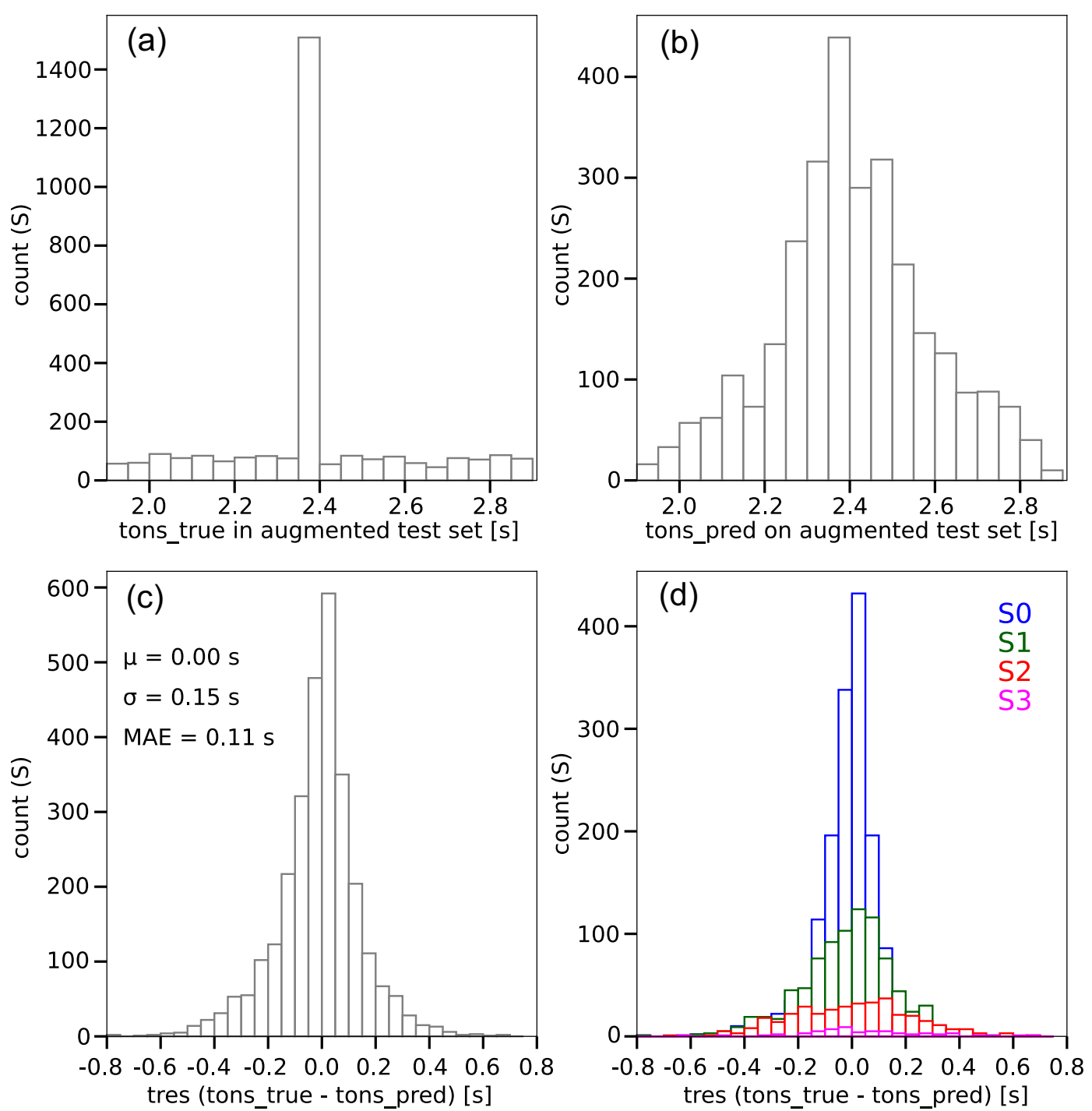

Figure 12. Evaluation of the optimized model trained for $S$-phase picking on the independent augmented test set of $1440 \times 2$ two-component waveform samples. Subplots are similar as in Fig. 11.

Fig. 3). The resulting time uncertainty is asymmetric and inversely proportional to the slope of the mean predicted probability curve around tpred. That is, the steeper the decrease/increase in the predicted probability before/after tpred, the lower the time uncertainty, as one might intuitively expect from an abrupt, easy-to-pick change from noise to signal. Based on these estimated pick uncertainties, we defined the weighting class scheme for the phases $P$ and $S$ shown in Table 3, which was adapted from (Sippl et al. 2013).

\section{RESULTS}

\subsection{Phase detection model optimization}

Fig. 4 summarizes how the six main hyperparameters in the phase detection model evolved over the 1000 optimization trials attempted. For the majority of the main hyperparameters, the most frequently sampled hyperparameter value (higher histogram bins) did not coincide with the value that led to the highest model accuracy found after 1000 trials (shaded histogram bin), although this correlation was observed for the number of convolutional layers and frac_pre_P.
Remarkably, as can be seen from the subplots in Fig. 5, the best hyperparameter values or the most frequently sampled values (when both did not coincide) formed clusters at several times (around trials $150,260,400,590$ and 800) during the optimization. This suggests that the same set of hyperparameter values was systematically found to be effective in producing highly accurate models during the optimization process, while the rest of the hyperparameters in the network were probably still being adjusted.

The best-performing hyperparameter values indicate that models in which the convolutional block was considerably deeper (five convolutional layers) than the dense block (only one or two dense layers) proved to be more accurate. Furthermore, models that learned from band-pass filtered data reached lower training accuracy in comparison to when high-pass filter or no filter was applied, with the latter approach performing the best. Thus, using the whole frequency content proved to be more effective for model training than removing very long period background seismic noise or substantially limiting the frequency content. We note here that quite a few of the data are from short-period seismometers, hence inherently high-pass filtered $(>1 \mathrm{~Hz})$, which could account for the most frequently sampled value of pre mode. 
(a)

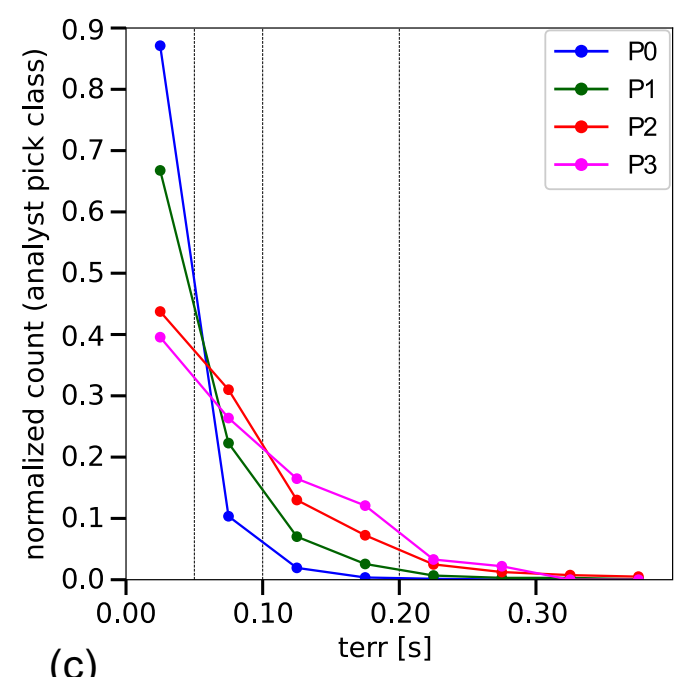

(c)

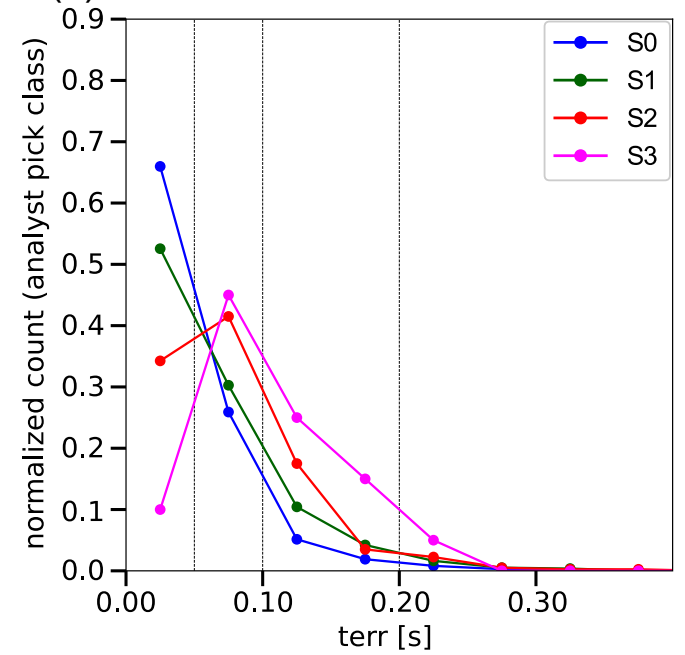

(b)
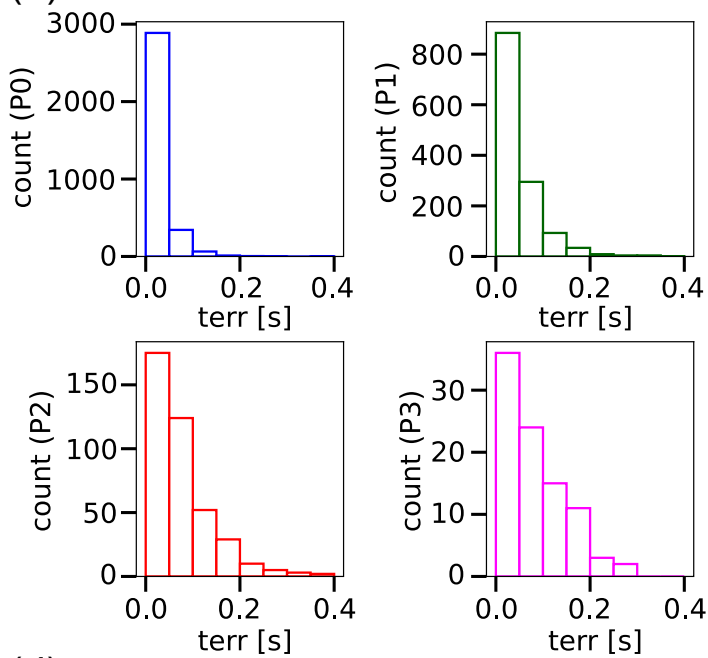

(d)
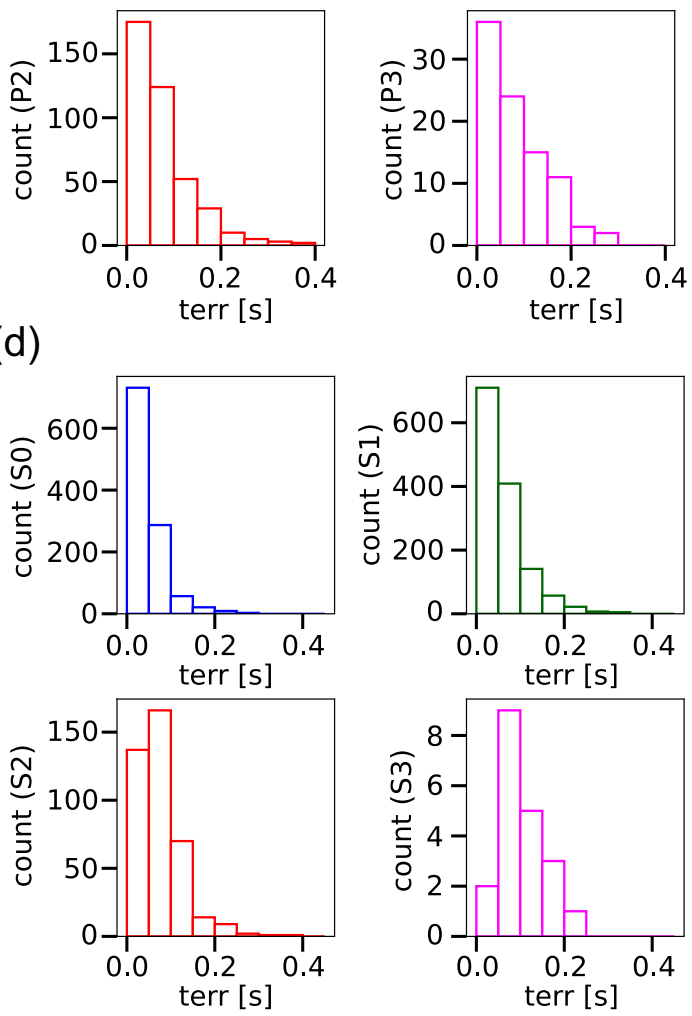

Figure 13. (a), (c) Normalized distribution of analyst P- and S-pick quality classes over the range of predicted time onset errors (terr in Table 3). Time errors were predicted on the augmented test sets, so analyst quality classes are counted twice. Points defining each coloured curve are plotted at the centre of the histogram bins corresponding to each pick class distribution. Dotted vertical black lines mark the time confidence interval limits used in the weighting scheme to assign the predicted pick classes (Table 3). (b), (d) Histograms (bin size $=0.05$ [s]) showing disaggregated distributions of analyst P- and S-pick classes shown in (a) and (c), respectively.

Another important constraint in the optimization process seems to be the amount of data available for the network to learn, since longer window samples (480-500 s) clearly led to more correctly predicted phases. $P$ phases were more accurately predicted when the sample windows include a relatively larger portion of noise ( 70 per cent) compared to signal, whereas $S$ phases were better predicted from windows containing similar amount of noise relative to signal (50 per cent).

Table 1 lists the best-performing values found for each of the optimized hyperparameters, which were used to train the best phase detection model architecture outlined in Fig. 6.

\subsection{Predicted phase classes compared to analyst labels}

Figs S2(a) and (b) show that the accuracy of the best phase detection model smoothly increased on the training set and reached 0.986 after 35 epochs, when the early stopping condition stopped the training. The training loss progressively decreased and reached
0.044 at the end of the training. The accuracy and loss calculated on the validation set followed a similar behavior to the training curves, although showing overall higher accuracy and lower loss due to the use of dropout regularization during training. Dropout disables a certain number of layer units, therefore some information about the input samples is lost and the network attempts to learn based on incomplete data representations. However, during validation all the units are available, thus the network uses its full computational power.

The confusion matrix in Fig. 7(c) shows the overall performance of the best trained model in classifying the classes of the independent test set of 4320 samples. The cells in this matrix are filled with the predicted classes, which correspond to the highest of the three-class probabilities returned by the model for each sample. An overall very high ratio (0.99) of correct predictions is observed for the three classes, as can be seen from the numbers in the matrix diagonal. Fig. S3 show waveform examples of these correctly predicted P-, S- and N-class samples. 




Figure 14. Automatically derived earthquake catalogues in northern Chile, from which two independent test sets of three-component samples were used to evaluate the DeepPhasePick performance. A first test set of 228230 samples was extracted from events occurring between 2013-06-01 and 2014-02-28 (T1: red circles; Sippl et al. 2018). A second test set of 1361544 samples was taken from events occurring between 2014-03-01 and 2014-12-31 (T2: green circles; Soto et al. 2019). Window samples obtained from $\mathrm{T} 1$ and $\mathrm{T} 2$ were registered by the 21 and 82 stations shown on the map. Focal mechanisms (beachballs) and epicentres (stars) of the Iquique M8.1 main shock, its largest M6.6 foreshock and its largest M7.6 aftershock (Hayes et al. 2014) are plotted for reference in orange, yellow and brown colour, respectively.

Of the few misclassified samples, only three correspond to $S$ phases predicted as $P$, whereas no $P$ phases were mispredicted as $S$, indicating that the network has a high capability to discriminate between both classes. Fig. S4 shows one of these misclassified $S$ phases, where the pick made by the analyst was somewhat earlier than the most impulsive change in amplitude. This could be one possible source of misclassification between the two phases, since it may have led to the network misinterpreting the sample as a $\mathrm{P}$ phase.
The remaining mispredictions correspond to either actual $P$ or $S$ phases predicted as noise (24 samples) or presumed noise predicted as $P$ or $S$ phases (21 samples). From the samples in the former group, the majority of the $P(90$ per cent) and $S(\sim 71$ per cent $)$ samples were assigned pick weights higher or equal than $1(<75$ per cent confidence) by analysts (Fig. 7e). This is indicative of waveforms with relatively low signal-to-noise ratio (SNR), as can be seen from the examples of mispredicted $P$ and $S$ phases shown in Figs 8(a) and (b), which may cause the model misclassification. In the latter 

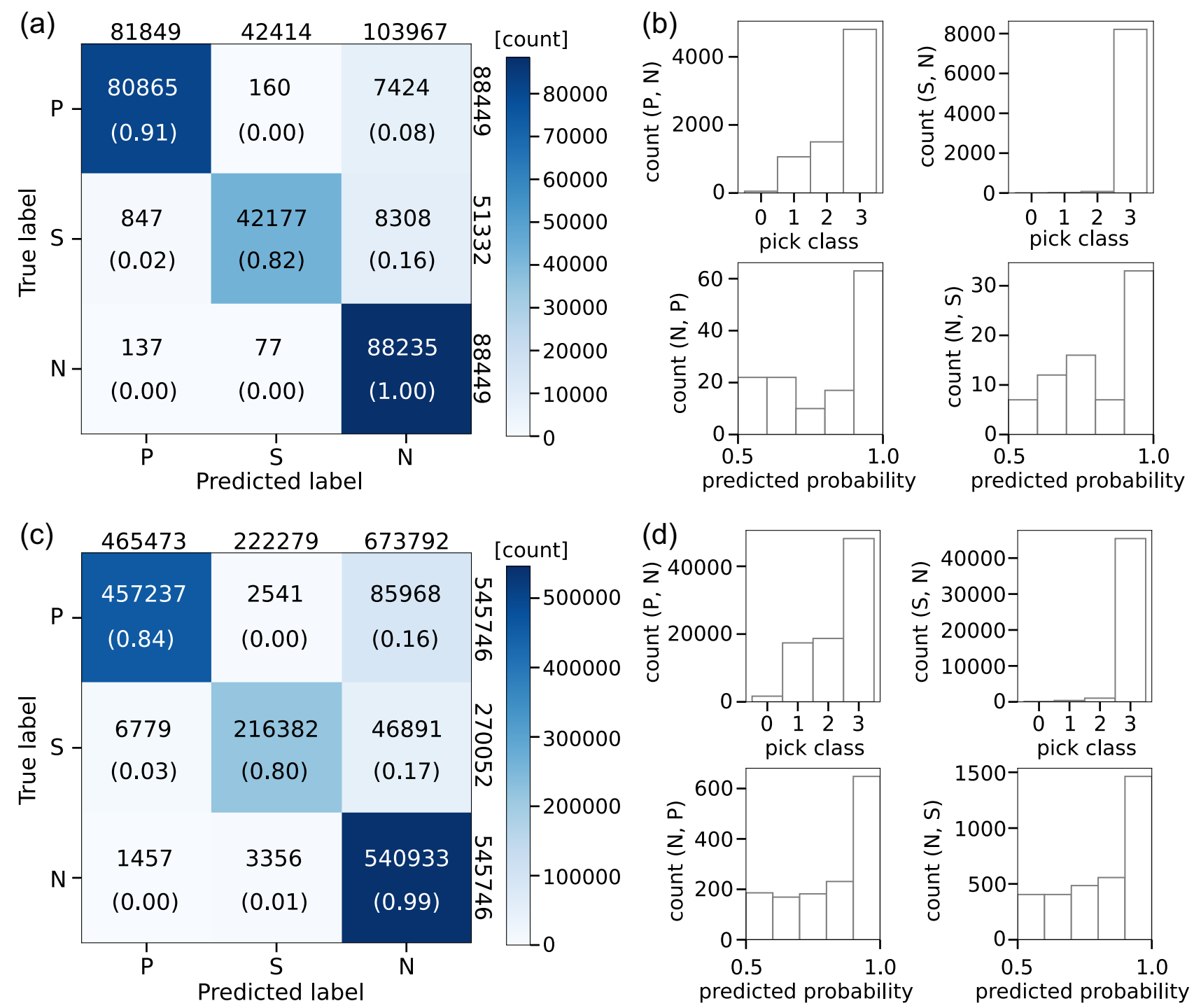

Figure 15. Results from the evaluation of the best model trained for phase detection on independent test sets taken from automatically derived earthquake catalogues T1 (a-b) and T2 (c-d). (a), (c) Confusion matrices. (b), (d) disaggregated histograms of incorrectly predicted $P$ or $S$ phases as noise (upper subplots) and presumably misclassified noise samples as $P$ or $S$ phases (lower subplots). (P, N) means $P$-phase samples predicted as Noise, and similarly for other cells in the confusion matrix.

group, a significant majority of the noise samples were predicted as signal with relatively high probability. In fact, of these, 87.5 per cent of the $P$ and $\sim 77$ percent of the $S$ predictions had a probability higher than 0.7 , with 75 per cent of the $P$ and 38 percent of the $S$ predictions having a probability higher than 0.9 (Fig. 7e). The examples in Figs 9(a) and (b) demonstrate that these apparently mispredicted samples actually correspond to previously undetected phases, hence evidencing the powerful detection capacity of the model.

The detection capability of the trained model can be further assessed by calculating the precision and recall metrics. Precision, given by TP/(TP + FP), describes how well the model predicts a specific class, by comparing the correct predictions of the class (true positives: TP) with all the predictions made for that class (TP + FP; where FP means false positives). Recall is computed as TP/(TP + FN) and reports the ability of the model to identify all the samples of a given class, by comparing TP with all the samples of that class in the test set (TP + FN; where FN are false negatives).
Precision and recall vary between 0 and 1 , where higher values are associated with better performing models. In a precision-recall curve, precision is plotted against recall for different probability thresholds, that is the probability above which a sample is predicted to be of a certain class. The more the curve bends toward the point $(1,1)$, and therefore the larger the area under the curve (AUC), the better the model performance. Fig. 7(d) shows the precisionrecall curve obtained for the samples in the independent test set. As can be seen here the performance of the model in predicting both P- and S-class samples was very high, with the performance being slightly better for the $\mathrm{P}$ than for the $\mathrm{S}$ class. The model performance on N-class samples was somewhat lower. However, this calculated performance does not take into account any presumed $\mathrm{N}$-class samples where the model detected a real seismic signal. As previously discussed by Ross et al. (2018b), the probability threshold can be tuned so as to improve precision (reducing false positives) or recall (aiming at reducing missed detections), depending on the application. 

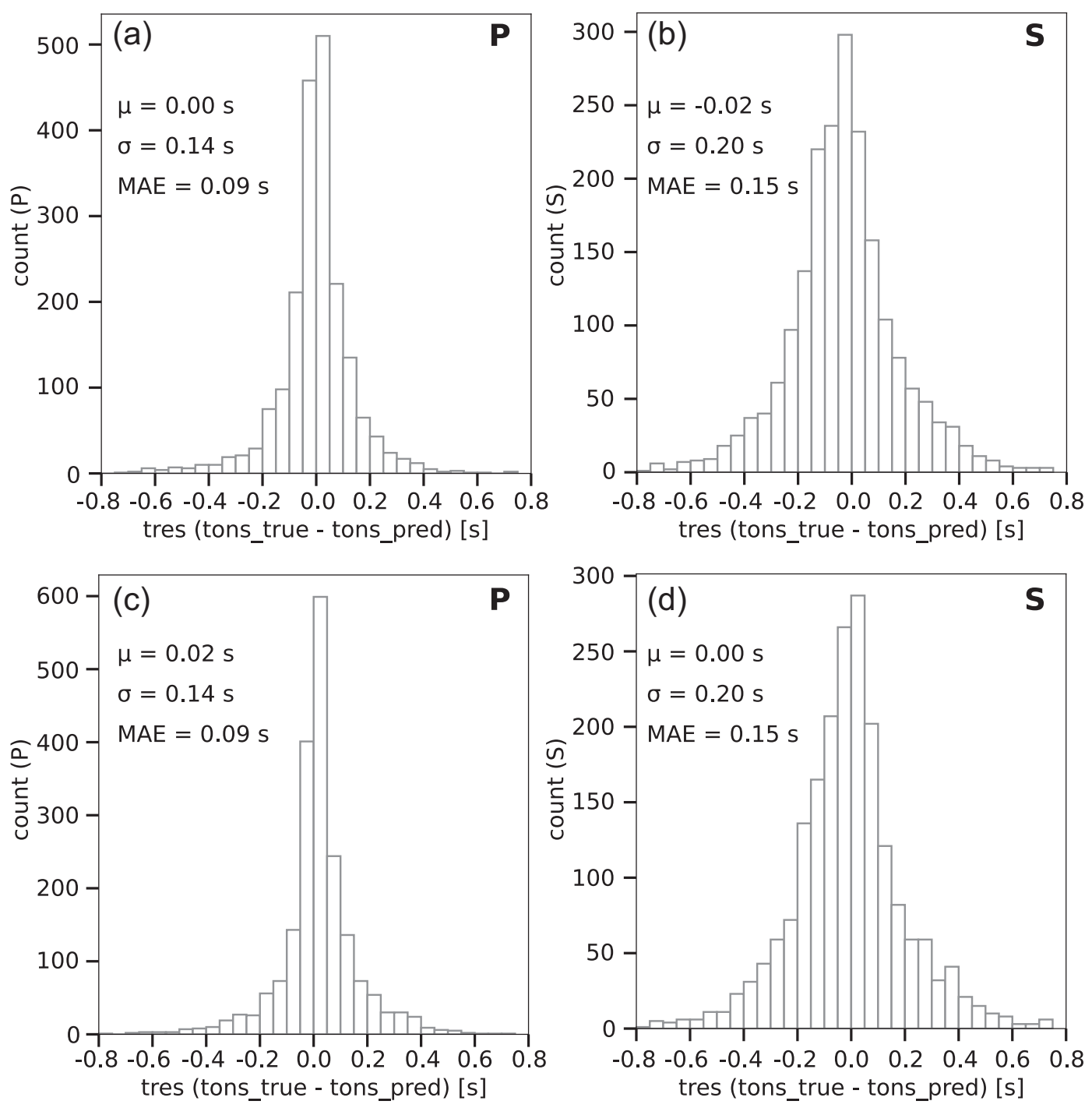

Figure 16. Results from the evaluation of the optimized model trained for $P$-phase picking on independent augmented test sets formed by $1000 \times 2$ samples taken from the automatically derived earthquake catalogues T1 (a and b) and T2 (c and d). (a) $P$-phase time residuals distribution for test samples taken from T1 (as in Fig. 11c). (b) $S$-phase time residuals distribution for test samples taken from T1 (as in Fig. 12c). (c) $P$-phase time residuals distribution for test samples taken from T2. (b) $S$-phase time residuals distribution for test samples taken from T2.

\subsection{Predicted phase time onsets compared to analyst picks}

Table 2 shows the best-performing hyperparameter values found during the optimization of the $P$ - and $S$-phase picking models. These parameters were used to train the best picking model architectures outlined in Figs 10(a) and (b).

At the end of the training, the best P-picking model reached higher accuracies (0.983 in training) than the accuracies of the S-picking model ( 0.972 in training). This is also reflected in the training and validation loss functions, which reached a lower value for $P$ phases (0.041 in training) than for $S$ phases (0.066 in training, Fig. S2). As previously explained, the MCD estimation of predicted phase time onsets and their uncertainties requires that the picking models are trained with dropout. For this reason the training and validation curves do not show the difference in accuracy and loss observed for the best phase detection model.

Figs 11 and 12 summarize the performance of the optimized models trained for picking $P$ and $S$ phases, when evaluated on the independent test sets of 2565 one-component and 1440 two-component samples respectively. In order to evaluate how the models perform in different possible picking scenarios, we used an augmented test set formed by two groups of samples: (1) the actual waveforms in the original test set, as defined by the optimized hyperparameters of the best phase detection model (win_size, frac_pre_P and frac_pre_S) and (2) artificial versions of the same waveforms, randomly shifted between -0.5 and $0.5 \mathrm{~s}$ around the true phase onsets made by the analysts.

The slightly higher accuracy reached by the $P$-phase picking model is reflected in the shape of the true and predicted time onset distributions, which more closely resemble each other for the $P$-phase (Figs 11a and b) compared to the $S$-phase (Figs 12a and b). Consequently, a narrower distribution of time residuals was obtained for $P$ phases compared to $S$ phases (Figs $11 \mathrm{c}$ and 12c). Overall, however, the time residual distributions for both $P$ and $S$ phases show a remarkable compliance with analyst picks. Based on the weighting class scheme defined in Table 3, we observe that a significant majority of $P$ - and $S$-phase onsets were predicted with lower uncertainties (pick quality classes 0 or 1 in Figs $11 \mathrm{~d}$ and 12d). Even though the distributions of all pick quality classes span over 


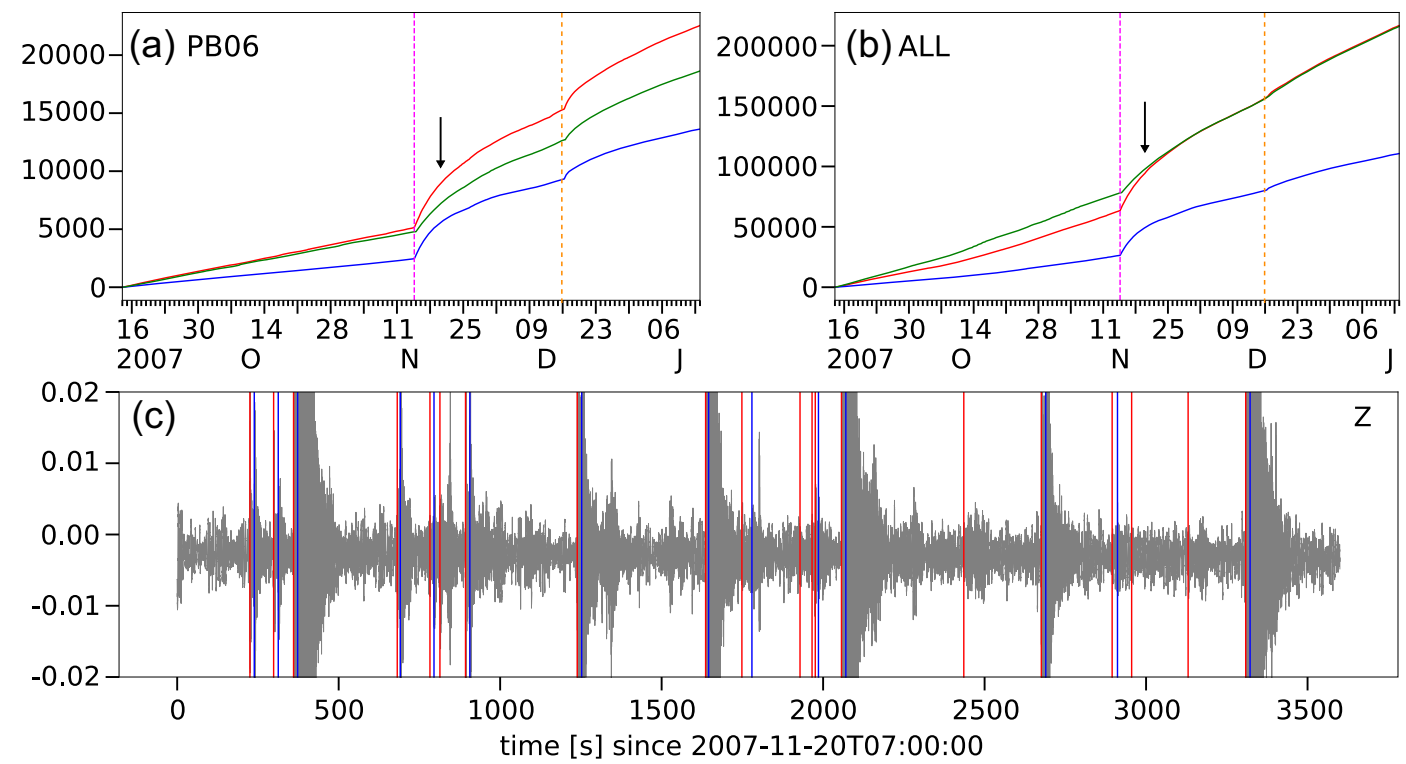

Figure 17. (a), (b) Cumulative number of $P$ (red curve) and $S$ (blue curve) phases predicted by DeepPhasePick ( $\left.p b_{-} P \_t h 1=p b_{-} S_{-} t h 1=0.98\right)$, and triggered detections by the STA/LTA algorithm (green curve) on continuous seismic data recorded at the PB06 station (a) and across all IPOC stations with available data (b). Cumulative curves are plotted for the time interval covering two months before up to two months after the 2007 M7.7 Tocopilla main shock (2007-11-14), which is marked as a magenta dashed line. An orange dashed line indicates the time of occurrence of a $M 7.1$ aftershock (2007-12-16) 50 km south of the main shock epicentre. (c) 1 -hr waveform snippet of PB06 station data, depicting the phases predicted by DeepPhasePick $\left(p b_{-} P_{-} t h 1=p b_{-} S_{-} t h 1=0.98\right)$ at the time indicated by the arrows in (a) and (b): red and blue lines represent $P$ and $S$ phases, respectively.

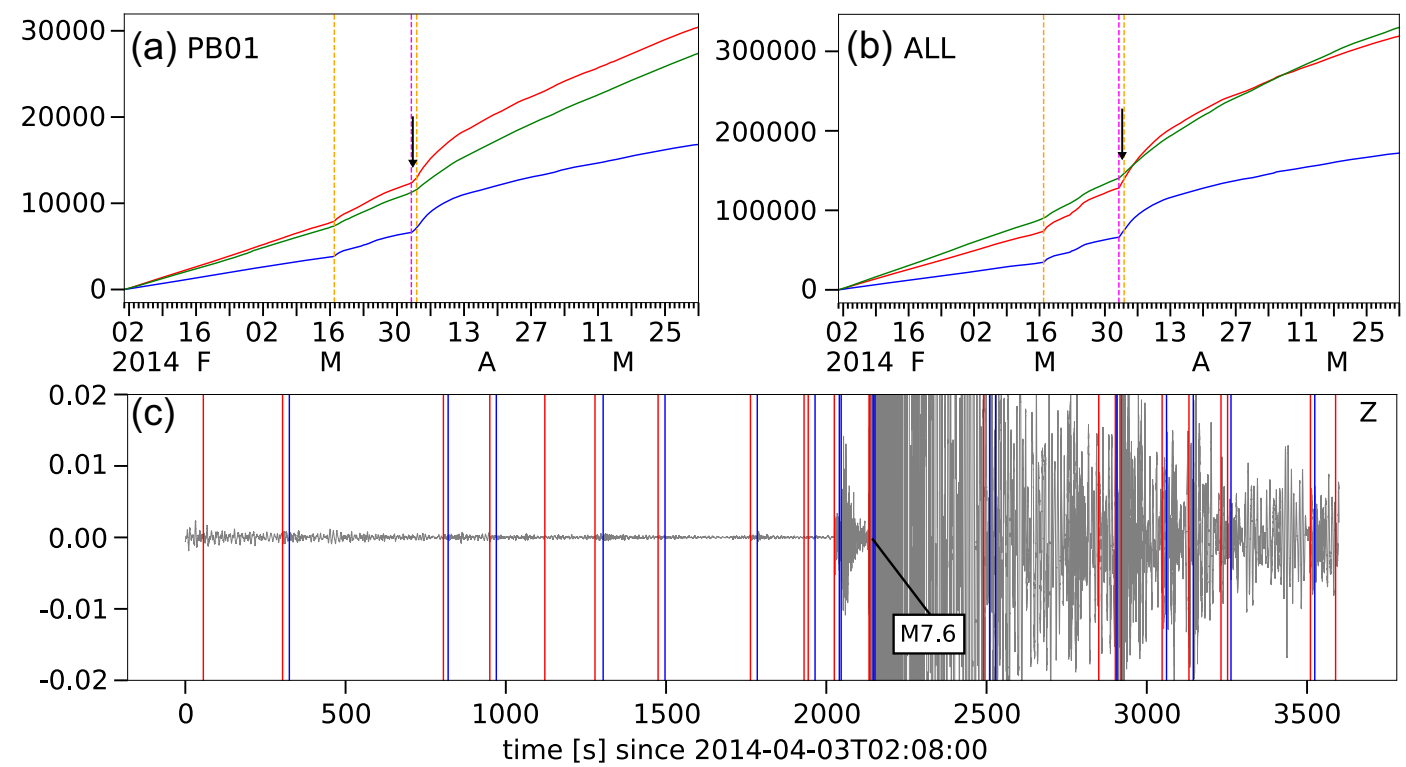

Figure 18. (a), (b) Cumulative number of $P$ (red curve) and $S$ (blue curve) phases predicted by DeepPhasePick ( $\left.p b_{-} P_{-} t h 1=p b_{-} S_{-} t h 1=0.98\right)$, and triggered detections by the STA/LTA algorithm (green curve) on continuous seismic data recorded at the PB01 station (a) and across all IPOC stations with available data (b). Cumulative curves are plotted for the time interval covering 2 months before up to two months after the 2014 M8.1 Iquique main shock (2014-04-01), which is marked as a magenta dashed line. Two additional orange dashed lines indicate the time of occurrence of the largest foreshock (M6.7; 2014-03-16) and aftershock $(M 7.6 ; 2014-04-03)$ in the Iquique sequence. (c) 1-hr waveform snippet of PB01 station data, depicting the phases predicted by DeepPhasePick ( $\left.p b_{-} P \_t h 1=p b_{-} S_{-} t h 1=0.95\right)$ at the time indicated by the arrows in (a) and (b): red and blue lines represent $P$ and $S$ phases, respectively.

a similar range of time residuals, the distribution is more flattened for higher uncertainties. This may suggest that picks predicted with lower uncertainties by the network were also easier to pick by the analyst (possibly due to a waveform with higher SNR), resulting in lower time residuals since both the network and analyst would have picked a similar onset. Conversely, picks that were harder to pick by the analyst (e.g. due to a waveform with lower SNR), were probably predicted with higher uncertainties by the network, which would lead to higher absolute residuals.

So as to investigate this correlation between predicted and analyst pick classes, we plotted the distribution of the analyst pick classes against the predicted time onset errors (terr) for both test sets (Fig. 13). Essentially, these distributions show that analyst classes do not always correlate with equivalent predicted 

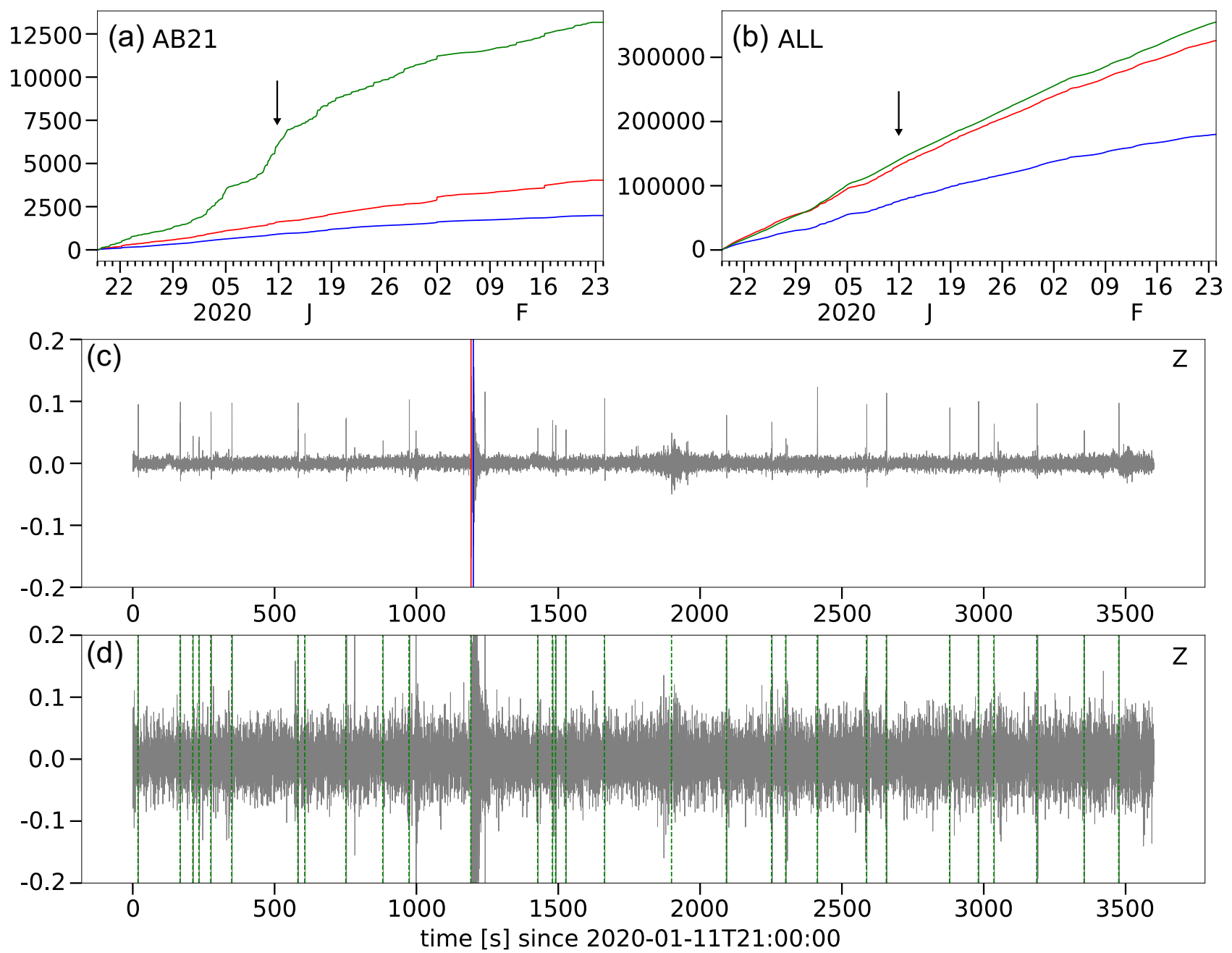

Figure 19. (a), (b) Cumulative number of $P$ (red curve) and $S$ (blue curve) phases predicted by DeepPhasePick ( $\left.p b_{-} P_{\_} t h 1=p b_{-} S_{-} t h 1=0.98\right)$, and triggered detections by the STA/LTA algorithm (green curve) on continuous seismic data recorded at the AB21 station (a) and across all Albanian stations with available data (b). Cumulative curves are plotted for the time interval between 2019-12-19 and 2020-02-24, during the aftershock sequence of the 2019 M6.4 Albania earthquake (2019-11-26). (c) 1-hr waveform snippet of AB21 station data, depicting the phases predicted by DeepPhasePick $\left(p b_{-} P_{-} t h 1=p b_{-} S_{-} t h 1=0.98\right)$ at the time indicated by the arrows in (a) and (b): red and blue lines represent $P$ and $S$ phases, respectively. (d) Green dashed lines depict the STA/LTA detections produced on the 1-hr waveform plotted in (c).

classes, but instead span the entire range of predicted time onset errors.

However, a couple of observations from the normalized distributions in Figs 13(a) and (c) may indicate that at least some degree of correlation does exist. First, lower-uncertainty analyst picks (classes 0 and 1) concentrate in predicted lower-error intervals (terr $<0.05$ ) in a considerably higher proportion, compared with higheruncertainty analyst picks (classes 2 and 3). Second, analyst picks of higher uncertainty decrease more gradually and rise above picks of lower uncertainty when terr $>0.05$.

\subsection{Evaluation on independent test sets from automatically derived earthquake catalogues}

We further analysed the performance of the best trained models of DeepPhasePick on two additional independent test sets. These test sets consisted of three-component window samples extracted from two earthquake catalogues recently published for the region where the 2014 M8.1 Iquique megathrust earthquake ruptured the northern Chilean subduction on 1 April 2014 (Fig. 14). The first catalogue (T1) contains 8278 events occurring in a time window of nine months before the Iquique main shock (between 2013-06-01 and 2014-02-28, Sippl et al. 2018), from which we extracted 228230 records (P: 88 449; N: 88 449; S: 51 332). The second catalogue (T2) includes 18963 events originating between 1 month before and 9 months after the main shock (between 2014-03-01 and 2014-12-31, Soto et al. 2019), from which we obtained 1361544 records (P: 545 746, N: 545 746, S: 270 052). Event records extracted from T1 and T2 were automatically picked by applying conventional stateof-the-art phase picking algorithms (Aldersons 2004; Di Stefano et al. 2006; Diehl et al. 2009), thus provide a valuable quantitative assessment when compared to DeepPhasePick predictions.

We first analysed the performance of the optimized model trained for phase detection. For this analysis, we used test sets formed by the entire set of samples available from catalogues T1 and T2. Fig. 15 summarizes the classification results from the evaluation of the model on both test sets. In the confusion matrices in Figs 15(a) and (c), we observe the highest ratio of correct predictions for noise samples, comparable to the evaluation performed on the test set extracted from data sets S1 and S2 (Fig. 7c). The percentage of 

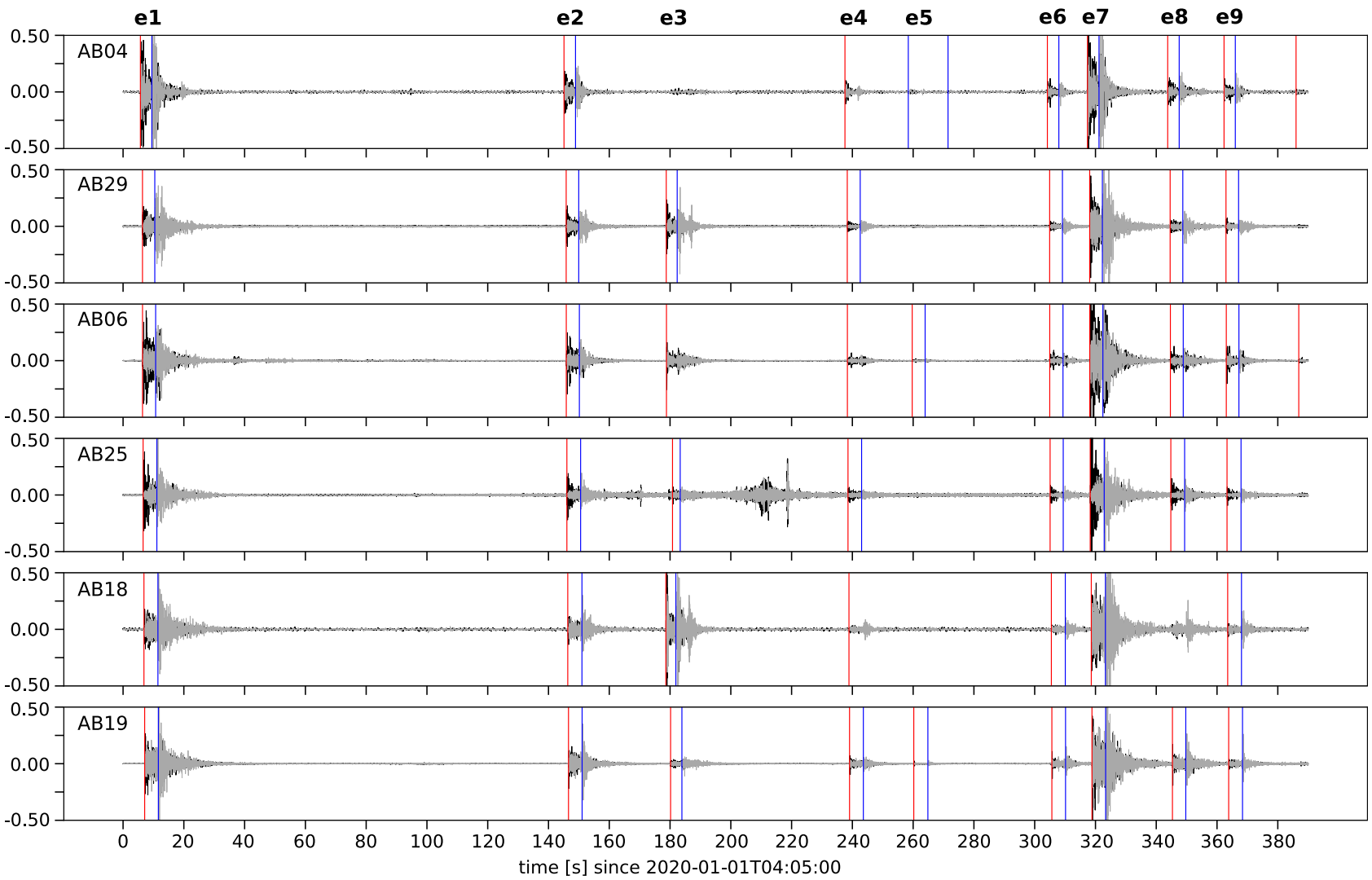

Figure 20. Example of multistation phase prediction made by DeepPhasePick $\left(p b_{-} P \_t h 1=p b_{-} S_{-} t h 1=0.98\right)$ on continuous seismic data in Albania. Red and blue lines mark predicted $P$ and $S$ phase time onsets, respectively. Horizontal north component (grey) is plotted on top of vertical component (black) in each station waveform. Several events are clearly detected across the station network.

correct classifications decreased by $8-15$ per cent for $\mathrm{P}$ samples and $17-19$ per cent for $\mathrm{S}$ samples.

From the mispredicted $\mathrm{P}$ and $\mathrm{S}$ records, only a small fraction corresponds to $P$ phase mispredicted as $\mathrm{S}$, or vice versa, compared to the number of $\mathrm{P}$ and $\mathrm{S}$ records which were predicted as noise. As we observed for the results in Fig. 7(c), the optimized model can mispredict $S$ phases as $P$ phases if the true $\mathrm{S}$ time onsets are somewhat earlier. Conversely, it is conceivable that the network classifies a $P$ phase as an $S$ phase if the true $\mathrm{P}$ time onset is late.

$P$ and $S$ phases predicted as noise correspond almost entirely to samples which were assigned automatic pick quality class 1 or worse ( $<75$ per cent confidence), with most of them being assigned the poorest pick quality class (class $3 ;<25$ per cent confidence) (upper subplots in Figs $15 \mathrm{~b}$ and d). As we have discussed, the classification of these hard-to-pick phases by the network appears to be challenging. Moreover, about half of the few apparently misclassified noise samples were predicted as $P$ or $S$ phases with a probability of at least 90 per cent (lower subplots in Figs $15 \mathrm{~b}$ and d), suggesting that these presumed noise records might actually represent phases previously undetected by the automatic detection procedure, similar to the examples shown in Fig. 9.

We next analysed the performance of the optimized models trained for $P$ - and $S$-phase picking, using test sets formed by 1000 $P$ - and $S$-phase samples randomly selected from $\mathrm{T} 1$ and $\mathrm{T} 2$ catalogues. As described in the previous section, the picking models were evaluated on augmented versions of these test sets.

Results from the picking predictions are summarized in Fig. 16. Time residual distributions, which are narrower for $P$ phases
(Figs 16a and c) compared to $S$ phases (Figs 16b and d), are observed in both test sets. Similar distribution shapes were obtained for the augmented test set derived from S1 and S2 data sets (Figs 11c and $12 \mathrm{c}$ ), hence evidencing that the trained picking models generalize effectively to different data sets.

\subsection{Prediction on continuous seismic waveform data}

After analysing the performance of DeepPhasePick with different independent sets, we used the best-performing trained models in the algorithm to carry out the phase detection and picking tasks on continuous seismic data recorded in two different tectonic environments. First, we applied DeepPhasePick on data which covers the time of two megathrust earthquake sequences that occurred in the northern Chile subduction zone (2007-11-14 M7.7 Tocopilla and 2014-04-01 M8.1 Iquique main shocks). In this case, the data used was recorded by network stations situated in desertic areas, far from cities, and therefore exposed to low background seismic noise. Next, DeepPhasePick was applied on seismic data that partly covers the aftershock series of a recent $M 6.4$ earthquake which occurred on 2019-11-26 near the port town in Durres, Albania, in a region of convergence between Adriatic and Eurasian plates. Here we used seismic data from a 30-station network deployed in the rupture area of the M6.4 earthquake, about one month after its occurrence. This station network covers an urban area, thus being subject to higher seismic-noise signals compared to northern Chile.

We numerically compared the prediction performance of DeepPhasePick in both tectonic regimes with the detections done by an 

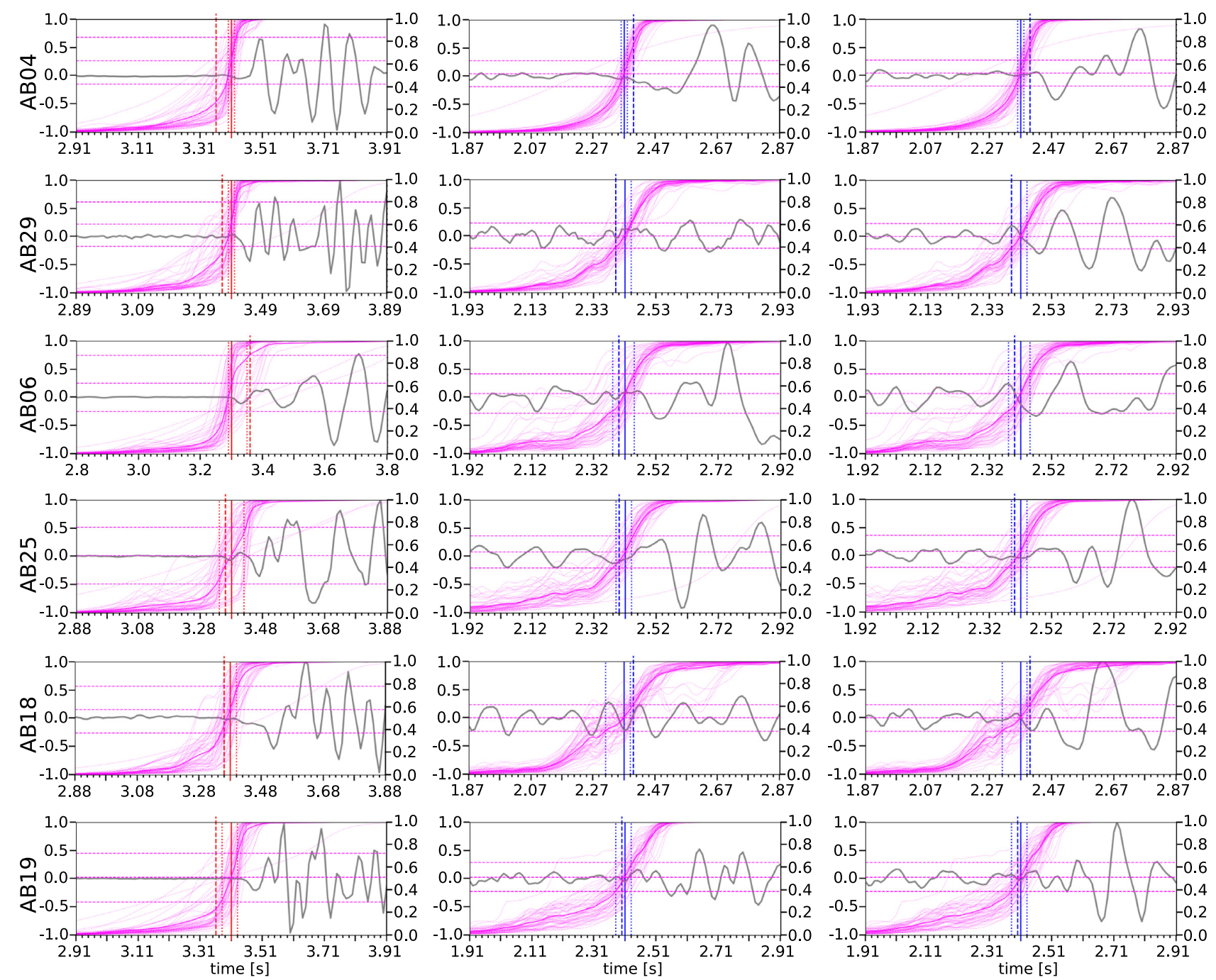

Figure 21. Estimation of time onset and associated uncertainty for predicted P (left-hand panel) and S (centre: E component, left: N component) phases of the event $\mathbf{e 1}$ in Fig. 20. Results from applying MCD method to each seismogram component are shown as in Fig. 3. Pick statistics for this event are reported in Table S2.

in-house developed STA/LTA trigger algorithm applied to all vertical component waveforms. For this test, we used STA and LTA window lengths of 1.0 and $20.0 \mathrm{~s}$; STA/LTA trigger and detrigger ratios of 8.0 and 1.5; and bandpass filter lower and upper corner frequencies of 2.0 and $10.0 \mathrm{~Hz}$.

\subsubsection{Performance in a lower-seismic noise region: northern Chile subduction zone}

The cumulative number of $P$ and $S$ phases predicted by DeepPhasePick on data from one representative as well as all available stations in northern Chile, depict an abrupt increase right after the occurrence of the 2007 M7.7 Tocopilla main shock (Figs 17a and b) and the 2014 M8.1 Iquique main shock (Figs 18a and b), corresponding to the beginning of the aftershock series of these large megathrust earthquakes. Less pronounced increments in cumulative predictions are observed at other times as well: after a $M 7.1$ aftershock occurring approximately one month following the Tocopilla main shock (Figs 17a and b), and after the occurrence of the largest foreshock (M6.7; 2014-03-16) and aftershock (M7.6; 2014-04-03) of the Iquique sequence (Figs 18a and b).
DeepPhasePick successfully captured a large majority of the various sized events occurring in the early post-seismic stage of the Tocopilla and Iquique main shocks (Figs 17c and 18c), which demonstrates the high resolution power of the algorithm. In particular, DeepPhasePick was capable of detecting $P$ and $S$ phases of several events occurring only a few minutes after the $M 7.6$ aftershock in the Iquique earthquake sequence (Fig. 18c).

\subsubsection{Performance in a higher-seismic noise region: Albania}

For most of the stations in northern Chile, the cumulative number of $P$ phases predicted by DeepPhasePick is comparable to the cumulative detections made by a classical STA/LTA algorithm (Figs S5 and S6), as one might expect from high-SNR seismic data. However, this does not hold true for several stations in Albania (Figs S7 and S8), which presumably recorded lower-SNR seismic data, though the overall cumulative number of predicted $P$ phases and STA/LTA detections across all the stations in this region is still comparable (Fig. 19b).

The discrepancy between the cumulative STA/LTA detections and DeepPhasePick predictions in the Albanian data arises mostly in two 
distinguishable scenarios. In the first scenario, cumulative STA/LTA detections greatly surpass $P$ - and $S$-phase predictions (e.g. stations AB05, AB12, or AB21). Figs 19(c) and (d) illustrate one example of this, where the STA/LTA algorithm detects numerous false positives in the noisy one-hour waveform, whereas DeepPhasePick predicts only one $P$ - and $S$-phase occurrence. In the second scenario, cumulative $P$-phase predictions are considerably higher than STA/LTA detections (e.g. stations AB10 or AB27, Figs S7 and S8). In this case, presumed false positive predicted by DeepPhasePick could be discarded, for instance, if they are not detected in a minimum number of stations when applying a phase associator algorithm.

In spite of eventual mispredictions generated due to noisy data, DeepPhasePick is generally able to detect multiple events across a station network. One example of multistation prediction is shown in Fig. 20, where six events are well detected in at least five stations within a 400-s window. Fig. 21 shows the corresponding time onsets for one of these events, generated by applying the MCD method in the picking stage. Similar results for two more events shown in Fig. 20 are presented in Figs S9 and S10. From the statistics of the picks predicted for the three events shown in Figs 21, S9 and S10, which are reported in Tables S2, S3 and S4, we observe that the difference between the preliminary phase time onsets (tons_prelim) obtained in the phase detection stage and the refined ones (tons_pred) computed in the picking stage can be up to $0.13 \mathrm{~s}$ for $P$ phases and up to $0.17 \mathrm{~s}$ for $S$ phases.

\section{DISCUSSION}

We have demonstrated that DeepPhasePick can be successfully applied to accurately detect and pick $P$ and $S$ phases originating from local earthquakes. The accurate predictions produced by DeepPhasePick result from the highly optimized set of hyperparameters defining its convolutional and recurrent deep neural networks trained for the tasks of seismic phase detection and picking, respectively. The systematic optimization process implemented aimed at compensating the limited seismic data used for training the models.

Results from the phase detection model optimization give us clues on the network architecture as well as input seismic samples characteristics that make the model perform more efficiently. First, the optimization indicates that the network detection capacity is enhanced by using a deeper block of convolutional layers. This can be explained by the fact that the model's ability to learn patterns from the input data, as well as the model's capacity to generalize so as to identify those patterns in new unseen data, are usually enhanced by adding convolutional layers to a $\mathrm{CNN}$-based model. A large number of dense layers in the network, which aim at learning nonlocal relationships in the input data, does not seem to be required for improving network performance. Secondly, the network learns features in $P$ and $S$ phases more effectively when it is fed with relatively long input seismic windows which have not been filtered. Longer windows may ease the extraction of relevant seismic features in order to better discriminate between $P$ and $S$ phases and identify noise, particularly if very long-period background seismic noise is present. Interestingly, the network detects $P$ phases better when sample windows contain a larger fraction of noise prior to a shorter portion of signal, in a way that resembles how analysts commonly identify this phase. Unlike $P$ phases, optimized detection of $S$ phases is obtained on sample windows consisting of a similar fraction of noise relative to signal, which may imply that the model learns patterns from features such as the $\mathrm{S}$ coda in order to recognize this phase.
Predictions performed on seismic samples from two independent test sets show that DeepPhasePick is capable of recognizing manually as well as automatically picked $P$ and $S$ phases with high accuracy, although it decreases for lower-quality automatic picks. These results also demonstrate that DeepPhasePick predicts phase time onsets which are comparable to those picked by analysts, as can be seen from the narrow time residual distributions in Figs 11(c) and 12(c). These residuals are comparable to those obtained in previous studies that implemented a deep learning-based picking approach (Ross et al. 2018a; Zhu \& Beroza 2018), even though a much smaller data set was used for training DeepPhasePick.

In particular, we compared the performances of DeepPhasePick and the recently published attention mechanism-based EQTransformer model (Mousavi et al. 2020c) on two different test sets. The first test set includes all the 2853 correctly classified $P$ - and $S$-phase samples from the phase detection evaluation (Fig. 7). The second test set is formed by a random selection of 1000 event samples (1000 P-phase and $1000 S$-phase samples) taken from the STEAD test set (Mousavi et al. 2019a), on which EQTransformer was evaluated (Mousavi et al. 2020c). Since EQTransformer was trained using longer samples than DeepPhasePick, to perform this comparison we extended the samples in the first set by including additional seismic data taken from the corresponding sample traces. To ensure that the EQTransformer detection worked properly, samples in the STEAD test set were also extended by adding a 10 -s noise window before and after each sample. These noise windows were formed by concatenating 10 times the first and the last second of the sample amplitudes, respectively. We performed the detection and picking applying DeepPhasePick and EQTransformer directly on the resulting MiniSeed samples. The resulting time residuals between the analyst phase time onsets and the predicted onsets from both methods are shown in Figs S11(c)-(f) for the first test set and Fig. S12 for the second test set. These results were obtained by applying EQTransformer using the best-performing parameters detection_threshold $=0.1, P \_$threshold $=0.1, S \_$threshold $=0.1$, overlap $=0.9$ and batch_size $=100$ in the function mseed predictor (as explained in https://eqtransformer.readthedocs.io/) and applying DeepPhasePick using probability trigger threshold of $p b_{-} P \_t h 1$ $=p b_{-} S_{-} t h 1=0.90$. In the first test set, S-phase time residuals are slightly more concentrated towards negative values for EQTransformer compared to DeepPhasePick. This time-shift is also observed for the $P$ - and $S$-phase time residual distributions obtained by applying DeepPhasePick on the STEAD test set, although in the opposite direction. This divergence indicates that DeepPhasePick predicts earlier picks than EQTransformer, which may reflect the difference in the picking criterion applied by analysts who built both test sets. Alternatively, this could be related to the characteristics of the seismic signal that each model learns during the training. Reported metrics indicate that both models perform comparatively well on both test sets, with EQTransformer doing slightly better on the STEAD test set. This demonstrates that using a model with highly optimized hyperparameters, such as DeepPhasePick, is a good alternative approach when only a relatively small training data set is available.

Some degree of correlation was found between analyst pick classes and classes predicted by DeepPhasePick (see Fig. 13). We note here that phase-pick weighting made by analysts is not an exact measure, but usually based on experience and intuition. Hence we would not necessarily expect a perfect correlation, and the weighting provided by DeepPhasePick might in fact be more objective.

DeepPhasePick predicts $P$ - and $S$-phase time onsets which are at least comparable to the time onsets obtained by applying dedicated 
automatic picking algorithms such as $M P X$ (Aldersons 2004; Di Stefano et al. 2006) and Spicker (Diehl et al. 2009), which served to derive the high resolution catalogues for the Northern Chile region shown in Fig. 14. Since DeepPhasePick also computes uncertainties and weights associated to the predicted picks, it can be used as a compelling alternative to those methods. However, DeepPhasePick is not restricted for use in the northern Chilean subduction zone, where the seismic samples used in training the models were originated. The algorithm is also able to predict $\mathrm{P}$ and $\mathrm{S}$ phases from local earthquakes occurring in a different tectonic regime, as we demonstrated for seismic data recorded by a 30 -station network in a region in Albania (e.g. Fig. 20).

DeepPhasePick has been designed to internally share the knowledge learned from the optimization of the phase detection model to the phase picking models, allowing the algorithm to perform both tasks in a joint two-stage process. Consequently, DeepPhasePick can be applied directly on continuous seismic waveforms with minor pre-processing involved, so as to determine accurate $\mathrm{P}$ and $\mathrm{S}$ time arrivals that can then feed a phase associator algorithm in the next stage of an automatic earthquake location workflow. Even though the training and inference (Monte Carlo sampling) times of the LSTMs used in the picking stage are slow compared to the CNN used for phase detection, this is to some degree balanced by the fact that the LSTMs only pick the phases within short time windows that have been previously detected by the CNN. DeepPhasePick can also be used to only output the preliminary arrivals obtained from the detection stage, in case fast pick estimation is required. However pick uncertainties are only obtained from the picking stage. In this final stage the picks are substantially improved, as illustrated in Figs S11(a)-(d), which shows the results from applying DeepPhasePick on the correctly classified samples from the phase detection evaluation. We can see that, compared to the preliminary predicted $\mathrm{P}$ and $\mathrm{S}$ picks obtained in the detection stage (standard deviations of 0.14 and $0.23 \mathrm{~s}$ for $P$ and $\mathrm{S}$ phases, respectively), the distributions of time residuals narrow after the picking stage (standard deviations of 0.11 and $0.21 \mathrm{~s}$ for $P$ and $S$ phases, respectively), indicating a significant reduction in the picking error after the second stage.

Further improvement of DeepPhasePick can be addressed in future work, for instance by retraining the $\mathrm{CNN}$-based phase detection model with additional manually picked samples from a new region of interest. A simple way to achieve this upgrade would be to use the new data for applying techniques such as feature extraction and fine tuning on the optimized model here presented. Further, the RNNbased phase picking models can be retrained by using more than one shifted version of each available seismic record (see e.g. Ross et al. 2018a). This would provide a broader spectrum of learnable sequential data during the training, which might improve the picking models performance, though with the caveat that the computing time required to train the models would further increase.

\section{CONCLUSION}

The fast development in deep learning algorithms has made it possible to reach super-human performance levels in tasks involving huge amount of data, such as image classification and natural language processing. In this work, we leveraged this computational progress to develop DeepPhasePick, a novel two-stage algorithm for detection and picking of $P$ and $S$ seismic phases originating from local earthquakes. DeepPhasePick has been built based on highly optimized convolutional and recurrent deep neural network architectures trained for such tasks. In a first stage, DeepPhasePick reports probabilities of waveform samples belonging to three phase classes (P, S or Noise). Preliminary phase onsets obtained from these probabilities are refined in a second step, in which final time onsets and their associated uncertainty are obtained by applying the Monte Carlo Dropout regularization technique, as an approximation of Bayesian variational inference.

DeepPhasePick has proven capable of recognizing both manually and automatically picked $P$ and $S$ seismic phases with high accuracy. It can also predict phase time onsets, which are comparable to those picked by analysts or derived from conventional, dedicated automatic phase picking algorithms. The $P$ - and $S$-phase time onsets, as well as their associated uncertainties, predicted by DeepPhasePick from continuous seismic data can be directly used to feed a phase associator algorithm as part of an automatic earthquake location workflow.

\section{ACKNOWLEDGEMENTS}

HS was previously supported by a CONICYT-DAAD stipend (57144001) and currently by the HAzard and Risk Team (HART) initiative of the GFZ German Research Centre for Geosciences in collaboration with the Institute of GeoSciences, Energy, Water and Environment of the Polytechnic University Tirana, Albania and the Karlsruhe Institute of Technology, Germany. We thank the developers of Hyperopt library, as well as Tensorflow and Keras machine learning frameworks, on which DeepPhasePick architecture was built. We further thank the developers of Python packages Obspy (Beyreuther et al. 2010) and Matplotlib (Hunter 2007), as well as Generic Mapping Tools (Wessel et al. 2019), which were used to create the figures and maps presented in this work. We thank two anonymous reviewers for their comments that helped improve the manuscript.

\section{DATA AVAILABILITY}

For training DeepPhasePick models for phase detection and picking tasks, we used waveform earthquake data collected from networks ZB (Schurr et al. 1999), ZE (Haberland et al. 1996), 8F (Wigger et al. 2016), Y9 (Fuenzalida et al. 2013), CX (GFZ German Research Centre for Geosciences \& Institut des Sciences de 1'Univers-Centre National de la Recherche CNRS-INSU 2006) and GE (GEOFON Data Centre 1993) accessed via EIDA web services (e.g. https://geofon.gfz-potsdam.de/).

For the evaluation of the performance of DeepPhasePick in independent test sets built from automatically derived earthquake catalogues, we used waveform earthquake data taken from networks CX, IQ (Cesca et al. 2009), 3D (Asch et al. 2014) and GE, as well as from Chilean Seismological Network (C, C1) stations (Barrientos 2018) accessed via IRIS webservices (http://ds.iris.edu/SeismiQuery/), the MEJIPE temporary network deployed by FU Berlin (Salazar et al. 2013) accessed via EIDA web services, and a temporary network deployed by the Chilean ONEMI, DGF, and CSN institutions accessed from $\mathrm{CSN}$ upon request.

The performance of DeepPhasePick predictions was further evaluated on continuous waveform data taken from networks $\mathrm{CX}$ and 9K (Schurr et al. 2020).

The optimized DeepPhasePick models trained for phase detection and phase picking tasks, together with an example script that applies DeepPhasePick method for both tasks on continuous waveforms, will be available via the GitHub repository https:/github.com/hso toparada/DeepPhasePick. 


\section{REFER EN CES}

Abadi, M. et al., 2015. TensorFlow: Large-scale machine learning on heterogeneous systems, Retrieved from https://www.tensorflow.org/ (software available from tensorflow.org).

Aldersons, F., 2004. Toward a three-dimensional crustal structure of the dead sea region from local earthquake tomography, $P h D$ thesis, Tel-Aviv University, Israel.

Allen, R.V., 1978. Automatic earthquake recognition and timing from single traces, Bull. seism. Soc. Am., 68(5), 1521-1532.

Asch, G., Tilmann, F., Heit, B. \& Schurr, B., 2014. HART-PISAGUA Project Chile, GFZ Data Services. Other/Seismic Network, https://doi.org/10.1 4470/8Q7569558037.

Baer, M. \& Kradolfer, U., 1987. An automatic phase picker for local and teleseismic events, Bull. seism. Soc. Am., 77(4), 1437-1445.

Barrientos, S., 2018. The seismic network of Chile, Seismol. Res. Lett., 89(2A), 467-474.

Bergstra, J., Bardenet, R., Bengio, Y. \& Kégl, B., 2011. Algorithms for hyper-parameter optimization, Proc. Adv. Neural Inform. Process. Syst., 24, 2546-2554.

Bergstra, J. \& Bengio, Y., 2012. Random search for hyper-parameter optimization, J. Mach. Learn. Res., 13, 281-305.

Bergstra, J., Yamins, D. \& Cox, D., 2013a. Making a science of model search: hyperparameter optimization in hundreds of dimensions for vision architectures, Proc. Int. Conf. Mach. Learn., 30, 115-123.

Bergstra, J., Yamins, D. \& Cox, D., 2013b. Hyperopt: a Python library for optimizing the hyperparameters of machine learning algorithms, Proc. Python Sci. Conf., 12, 13-20.

Beyreuther, M., Barsch, R., Krischer, L., Megies, T., Behr, Y. \& Wassermann, J., 2010. Obspy: a Python toolbox for seismology, Seismol. Res. Lett., 81(3), 530-533.

Cesca, S., Sobiesiak, M., Tassara, A., Olcay, M., Günther, E., Mikulla, S. \& Dahm, T., 2009. The Iquique Local Network and PicArray, GFZ Data Services. Other/Seismic Network, https://doi.org/10.14470/vd070092.

Chollet, F., 2015. Keras, https://github.com/fchollet/keras.

Chollet, F., 2017. Xception: deep learning with depthwise separable convolutions, in Proceedings of the IEEE Conference on Computer Vision and Pattern Recognition, pp. 1251-1258.

Di Stefano, R., Aldersons, F., Kissling, E., Baccheschi, P., Chiarabba, C. \& Giardini, D., 2006. Automatic seismic phase picking and consistent observation error assessment: application to the Italian seismicity, Geophys. J Int., 165(1), 121-134.

Diehl, T., Deichmann, N., Kissling, E. \& Husen, S., 2009. Automatic Swave picker for local earthquake tomography, Bull. seism. Soc. Am., 99(3), 1906-1920.

Dokht, R.M., Kao, H., Visser, R. \& Smith, B., 2019. Seismic event and phase detection using time-frequency representation and convolutional neural networks, Seismol. Res. Lett., 90(2A), 481-490.

Earle, P.S. \& Shearer, P.M., 1994. Characterization of global seismograms using an automatic-picking algorithm, Bull. seism. Soc. Am., 84, 366-376.

Folesky, J., Kummerow, J. \& Shapiro, S.A., 2018. Patterns of rupture directivity of subduction zone earthquakes in northern Chile, J. geophys. Res., 123, $10785-10796$.

Fuenzalida, A., Schurr, B., Lancieri, M., Sobiesiak, M. \& Madariaga, R., 2013. High-resolution relocation and mechanism of aftershocks of the 2007 Tocopilla (Chile) earthquake, Geophys. J Int., 194(2), 1216-1228.

Gal, Y. \& Ghahramani, Z., 2016a. Dropout as a Bayesian approximation: representing model uncertainty in deep learning, Proc. Int. Conf. Mach. Learn., 33, 1651-1660.

Gal, Y. \& Ghahramani, Z., 2016b. A theoretically grounded application of dropout in recurrent neural networks, Proc. Adv. Neural Inform. Process. Syst., 29, 1019-1027.

GEOFON Data Centre, 1993. GEOFON Seismic Network. Deutsches GeoForschungsZentrum GFZ, Other/Seismic Network, https://doi.org/10.1 4470/TR560404.

GFZ German Research Centre for Geosciences \& Institut des Sciences de l'Univers-Centre National de la Recherche CNRS-INSU, 2006. IPOC Seismic Network. Integrated Plate boundary Observatory Chile - IPOC, Other/Seismic Network, https://doi.org/10.14470/PK615318.
Gibbons, S.J. \& Ringdal, F., 2006. The detection of low magnitude seismic events using array-based waveform correlation, Geophys. J Int., 165(1), $149-166$.

Goodfellow, I., Bengio, Y. \& Courville, A., 2016. Deep Learning, Vol. 1, MIT Press.

Haberland, C., Rietbrock, A., Asch, G. \& Chong, G., 1996. The ANCORP Seismic Network, GFZ Data Services. Other/Seismic Network, https: //doi:10.14470/MR6441682066.

Harris, D.B., 1991. A waveform correlation method for identifying quarry explosions, Bull. seism. Soc. Am., 81, 2395-2418.

Harris, D.B., 1997. Waveform correlation methods for identifying populations of calibration events, in Proceedings of the 19th Annual Seismic Research Symposium on Monitoring a CTBT, September 23-25, pp. 604614.

Harris, D.B., 2001. Subspace techniques for detecting repeating events, poster, SSA 2001 Annual Meeting, San Francisco, CA, 18-20 April 2001 [Abstract], Seismol. Res. Lett., 72(2), 245.

Harris, D.B. \& Dodge, D.A., 2011. An autonomous system for grouping events in a developing aftershock sequence, Bull. seism. Soc. Am., 101, 763-774.

Harris, D.B. \& Paik, T., 2006. Subspace detectors: efficient implementation, Tech. Rep., Internal Report UCRL-TR-223177, Lawrence Livermore National Laboratory, 2006.

Hayes, G.P., Herman, M.W., Barnhart, W.D., Furlong, K.P., Riquelme, S., Benz, H.M., et al., 2014. Continuing megathrust earthquake potential in Chile after the 2014 Iquique earthquake, Nature, 512, 295-298.

Hinz, T., Navarro-Guerrero, N., Magg, S. \& Wermter, S., 2018. Speeding up the hyperparameter optimization of deep convolutional neural networks, Int. J. Comput. Intell. Applicat., 17(2), 1850008.

Hochreiter, S. \& Schmidhuber, J., 1997. Long short-term memory, Neural Comput., 9, 1735-1780.

Huang, H., Xu, W., Meng, L., Burgmann, R. \& Baez, J.C., 2017. Early aftershocks and afterslip surrounding the $2015 \mathrm{Mw} 8.4$ Illapel rupture, Earth Planet. Sci. Lett., 457, 282-291.

Hubel, D.H. \& Wiesel, T.N., 1959. Receptive fields of single neurons in the cat's striate cortex, J. Physiol., 148, 574-591.

Hubel, D.H. \& Wiesel, T.N., 1962. Receptive fields, binocular interaction, and functional architecture in the cat's visual cortex, J. Physiol., 160, $106-154$.

Hunter, J.D., 2007. Matplotlib: a 2D graphics environment, Comput. Sci. Eng., 9, 90-95.

Igarashi, T., Matsuzawa, T. \& Hasegawa, A., 2003. Repeating earthquakes and interplate aseismic slip in the northeastern Japan subduction zone, $J$. geophys. Res., 108(B5), 2249.

Ioffe, S. \& Szegedy, C., 2015. Batch normalization: accelerating deep network training by reducing internal covariate shift, Proc. Int. Conf. Mach. Learn.,37,448-456.

Kato, A. \& Igarashi, T., 2012. Regional extent of the large coseismic slip zone of the $2011 \mathrm{Mw} 9.0$ Tohoku-Oki earthquake delineated by on-fault aftershocks, Geophys. Res. Lett., 39, L15301.

Kingma, D.P. \& Ba, J., 2014. Adam: a method for stochastic optimization, Preprint at ArXiv, http://arxiv.org/abs/1412.6980.

Kriegerowski, M., Petersen, G.M., Vasyura-Bathke, H. \& Ohrnberger, M., 2019. A deep convolutional neural network for localization of clustered earthquakes based on multistation full waveforms, Seismol. Res. Lett., 90(2A), 510-516.

Krizhevsky, A., Sutskever, I. \& Hinton, G., 2012. ImageNet classification with deep convolutional neural networks, Proc. Adv. Neural Inform. Process. Syst., 25, 1097-1105.

LeCun, Y., Bengio, Y. \& Hinton, G., 2015. Deep learning, Nature, 521(7553), 436-444.

LeCun, Y., Boser, B., Denker, J.S., Henderson, D., Howard, R.E., Hubbard, W., et al., 1990. Handwritten digit recognition with a backpropagation network, Proc. Adv. Neural Inform. Process. Syst., 2, 396-404.

LeCun, Y., Bottou, L., Bengio, Y. \& Haffner, P., 1998. Gradientbased learning applied to document recognition, Proc. IEEE, 86, 2278-2324. 
Maceira, M., Rowe, C.A., Beroza, G. \& Anderson, D., 2010. Identification of low-frequency earthquakes in non-volcanic tremor using the subspace detector method, Geophys. Res. Lett., 37, L06303.

McBrearty, I.W., Delorey, A.A. \& Johnson, P.A., 2019. Pairwise association of seismic arrivals with convolutional neural networks, Seismol. Res. Lett., 90(2A), 503-509.

Moseley, B., Markham, A. \& Nissen-Meyer, T., 2018. Fast approximate simulation of seismic waves with deep learning, Preprint at ArXiv, http://arxiv.org/abs/1807.06873.

Mousavi, S.M. \& Beroza, G.C., 2020a. A machine-learning approach for earthquake magnitude estimation, Geophys. Res. Lett., 47, e2019GL085976, doi:10.1029/2019GL085976.

Mousavi, S.M. \& Beroza, G.C., 2020b. Bayesian-deep-learning estimation of earthquake location from single-station observations, IEEE Trans. Geosci. Remote Sens., 58(11), 8211-8224.

Mousavi, S.M., Ellsworth, W.L., Zhu, W., Chuang, L.Y. \& Beroza, G.C., 2020c. Earthquake transformer - an attentive deep-learning model for simultaneous earthquake detection and phase picking, Nat. Commun., 11(1), 1-12.

Mousavi, S.M., Sheng, Y., Zhu, W. \& Beroza, G. C., 2019a. STanford EArthquake Dataset (STEAD): a global data set of seismic signals for AI, IEEE Access, 7, 179464-179476.

Mousavi, S.M., Zhu, W., Ellsworth, W.L. \& Beroza, G.C., 2019b. Unsupervised clustering of seismic signals using deep convolutional autoencoders, IEEE Trans. Geosci. Remote Sens., 16(11), 1693-1697.

Mousavi, S.M., Zhu, W., Sheng, Y. \& Beroza, G.C., 2019c. CRED: a deep residual network of convolutional and recurrent units for earthquake signal detection, Scient. Rep., 9, 10267.

Nadeau, R.M. \& Johnson, L.R., 1998. Seismological studies at Parkfield VI: moment release rates and estimates of source parameters for small repeating earthquakes, Bull. seism. Soc. Am., 88(3), 790-814.

Nair, V. \& Hinton, G., 2010. Rectified linear units improve restricted Boltzmann machines, Proc. Int. Conf. Mach. Learn., 27, 807-814.

Panakkat, A. \& Adeli, H., 2009. Recurrent neural network for approximate earthquake time and location prediction using multiple seismicity indicators, Comp.-Aided Civil Infrastruct. Eng., 24, 280-292.

Pardo, E., Garfias, C. \& Malpica, R., 2019. Seismic phase picking using convolutional networks, IEEE Trans. Geosci. Remote Sens., 57(9), 70867092.

Peng, Z. \& Zhao, P., 2009. Migration of early aftershocks following the 2004 Parkfield earthquake, Nat. Geosci., 2, 877-881.

Perol, T., Gharbi, M. \& Denolle, M., 2018. Convolutional neural network for earthquake detection and location, Sci. Adv., 4(2), e1700578.

Ross, Z.E., Meier, M.-A. \& Hauksson, E., 2018a. P wave arrival picking and first-motion polarity determination with deep learning, J. geophys. Res., 123(6), 5120-5129.

Ross, Z.E., Meier, M.-A., Hauksson, E. \& Heaton, T.H., 2018b. Generalized seismic phase detection with deep learning, Bull. seism. Soc. Am., 108(5A), 2894-2901.

Ross, Z.E., Trugman, D., Hauksson, E. \& Shearer, P.M., 2019a. Searching for hidden earthquakes in Southern California, Science, 364, 767-771.

Ross, Z.E., Yue, Y., Meier, M.-A., Hauksson, E. \& Heaton, T.H., 2019 b. PhaseLink: a deep learning approach to seismic phase association, $J$. geophys. Res., 124, 856-869.

Rumelhart, D., Hinton, G. \& Williams, R., 1986. Learning representations by back-propagating errors, Nature, 323, 533-536.

Saad, O.M. \& Chen, Y., 2020. Earthquake detection and P-wave arrival time picking using capsule neural network, IEEE Trans. Geosci. Remote Sens., 59(7), 6234-6243.

Salazar, P., Wigger, P., Bloch, W., Asch, G., Shapiro, S.A. \& Kummerow, J., 2013. MEJIPE, GFZ Data Services. Other/Seismic Network, https: //doi.org/10.7914/SN/8G_2013.

Scharf, L.L. \& Friedlander, B., 1994. Matched subspace detectors, IEEE Trans. Signal Process., 42, 2146-2157.

Schurr, B., Asch, G., Rietbrock, A., Kind, R., Pardo, M., Heit, B. \& Monfret, T., 1999. Seismicity and average velocities beneath the Argentine Puna Plateau, Geophys. Res. Lett., 26(19), 3025-3028.
Schurr, B., Asch, G., Rosenau, M., Wang, R., Oncken, O., Barrientos, S., et al., 2012. The 2007 M7.7 Tocopilla northern Chile earthquake sequence: implications for along-strike and downdip rupture segmentation and megathrust frictional behavior, J. geophys. Res., 117(B5), doi:10.1029/2011JB009030.

Schurr, B., Dushi, E., Rietbrock, A. \& Duni, L., 2020. AlbACa Albanian Earthquake Aftershock Campaign, GFZ Data Services. Other/Seismic Network,

Schurr, B., Rietbrock, A., Asch, G., Kind, R. \& Oncken, O., 2006. Evidence for lithospheric detachment in the central Andes from local earthquake tomography, Tectonophysics, 415, 203-223.

Schuster, M. \& Paliwal, K.K., 1997. Bidirectional recurrent neural networks, IEEE Trans. Signal Process., 45(11), 2673-2681.

Shelly, D.R., Beroza, G.C. \& Ide, S., 2007. Non-volcanic tremor and lowfrequency earthquake swarms, Nature, 446, 305-307.

Shoji, D., Noguchi, R., Otsuki, S. \& Hino, H., 2018. Classification of volcanic ash particles using a convolutional neural network and probability, Scient. Rep., 8(1), 8111-12.

Simard, D., Steinkraus, P.Y. \& Platt, J.C., 2003. Best practices for convolutional neural networks, Proc. Int. Conf. Doc. Anal. Recog., 7, 958-963.

Sippl, C., Schurr, B., Asch, G. \& Kummerow, J., 2018. Seismicity structure of the Northern Chile forearc from $>100,000$ double-difference relocated hypocenters, J. geophys. Res., 123, 4063-4087.

Sippl, C., Schurr, B., Yuan, X., Mechie, J., Schneider, F.M., Gadoev, M., et al., 2013. Geometry of the Pamir-Hindu Kush intermediate-depth earthquake zone from local seismic data, J. geophys. Res., 118, 1438-1457.

Sleeman, R. \& van Eck, T., 1999. Robust automatic P-phase picking: an online implementation in the analysis of broadband seismogram recordings, Phys. Earth Planet. Inter., 113, 265-275.

Soto, H., Sippl, C., Schurr, B., Kummerow, J., Asch, G., Tilmann, F., et al., 2019. Probing the northern Chile megathrust with seismicity: the 2014 M8.1 Iquique earthquake sequence, J. geophys. Res., 124, 12 935-12 954.

Srivastava, N., Hinton, G., Krizhevsky, A., Sutskever, I. \& Salakhutdinov, R., 2014. Dropout: a simple way to prevent neural networks from overfitting, J. Mach. Learn. Res., 15, 1929-1958.

Taigman, Y., Yang, M., Ranzato, M. \& Wolf, L., 2014. Deepface: closing the gap to human-level performance in face verification, in Proceedings of the 2014 IEEE Conference on Computer Vision and Pattern Recognition, 23-28 June 2014, Columbus, OH, USA, pp. 1701-1708.

Van Trees, H.L., 1968. Detection, Estimation and Modulation Theory, John Wiley and Sons, Inc.

Wessel, P., Luis, J., Uieda, L., Scharroo, R., Wobbe, F., Smith, W.H.F. \& Tian, D., 2019. The Generic Mapping Tools Version 6, Geochem. Geophys. Geosyst., 20(11), 5556-5564.

Wigger, P., Salazar, P., Kummerow, J., Bloch, W., Asch, G. \& Shapiro, S., 2016. West-fissure- and atacama-fault seismic network (2005/2012). Deutsches GeoForschungsZentrum, GFZ. Other/Seismic Network, https: //doi:10.14470/3S7550699980.

Woollam, J., Rietbrock, A., Bueno, A. \& Angelis, S. D., 2019. Convolutional neural network for seismic phase classification, performance demonstration over a local seismic network, Seismol. Res. Lett., 90(2A), 491-502.

Zhang, X., Zhang, J., Yuan, C., Liu, S., Chen, Z. \& Li, W., 2020. Locating induced earthquakes with a network of seismic stations in Oklahoma via a deep learning method, Scient. Rep., 10(1), 1-12.

Zheng, J., Harris, J., Li, D. \& Al-Rumaih, B., 2020. SC-PSNET: a deep neural network for automatic P- and S-phase detection and arrival-time picker using 1C recordings, Geophysics, 85(4), U87-U98.

Zhou, Y., Yue, H., Kong, Q. \& Zhou, S., 2019. Hybrid event detection and phase-picking algorithm using convolutional and recurrent neural networks, Seismol. Res. Lett., 90(3), 1079-1087.

Zhu, W. \& Beroza, G.C., 2018. Phasenet: a deep-neural-networkbased seismic arrival-time picking method, Geophys. J Int., 216(1), 261-273.

Zhu, W., Li, X., Liu, C., Xue, F. \& Han, Y., 2020. An STFT-LSTM system for P-wave identification, IEEE Trans. Geosci. Remote Sens., 17(3), 519-523. 


\section{SUPPORTING INFORMATION}

Supplementary data are available at $G J I$ online.

Table S1. Precision, Recall and F1-score evaluation metrics at two probability thresholds in Fig. 7(b). Here F1 - score $=2 \times$ $\frac{\text { Precision } \times \text { Recall }}{\text { Precision }+ \text { Recall }}$

Table S2. Statistics of predicted picks shown in Fig. 21 for event e1 in Fig. 20.

Table S3. Statistics of predicted picks shown in Fig. S9 for event e7 in Fig. 20.

Table S4. Statistics of predicted picks shown in Fig. S10 for event e8 in Fig. 20.

Figure S1. $(\mathrm{a}-\mathrm{c})$ One representative earthquake waveform from Northern Chile, which contains the examples of predicted E, N, Z (east, north, vertical) sample windows shown in Fig. 3. Red and blue solid lines represent the predicted $P$ - and $S$-phase time onsets obtained in the phase picking stage. (d) Predicted probability timeseries for the Noise (N), $P$ - and $S$-phase classes obtained from the phase detection stage. Predicted probabilities are assigned at the centre of the moving window with length defined by the optimized hyperparameter win size (see Section 2.5). (e) Predicted probability time-series for the $P$ - and $S$-phase classes used in the picking stage. The $P$-phase time-series is shifted in time compared to the one in (d) due to the optimized hyperparameter value frac_pre_P (0.7). The S-phase time-series is not shifted since frac_pre_S is 0.5 (see Section 2.5). A grey dashed line indicates the probability threshold applied in the detection stage $\left(p b_{-} P_{-} t h 1=p b_{-} S_{-} t h 1=\right.$ $0.90)$. Predicted $P$ - and $S$-phase time onsets are shown as in (a-c).

Figure S2. ( $a$ and $b$ ) Evolution of the training and validation accuracy (a) and loss function (b) over the training epochs of the optimized model trained for phase detection. (c-f) Evolution of the training and validation accuracy and loss function over the training epochs of the optimized model trained for $P$-phase (c and d) and $S$-phase (e and f) picking.

Figure S3. Examples of correctly predicted P- (a), S- (b) and Nclass (c) samples in the test set of 4320 samples. Subplots are plotted the same way as in Fig. 8.

Figure S4. One example of S phase in the test set of 4320 samples, which was misclassified by the network as $P$-phase. Subplots are plotted the same way as in Fig. 8.

Figure S5. Cumulative number of $P$ (red curve) and $S$ (blue curve) phases predicted by DeepPhasePick $\left(p b_{-} P \_t h 1=p b_{-} S_{-} t h 1=\right.$ 0.98 ), and triggered detections by the STA/LTA algorithm (green curve) on continuous seismic data recorded by the stations with available data in northern Chile. Last subplot shows the combined results for all the stations in the network. Cumulative curves are plotted for the time interval between two months before and two months after the 2007 M7.7 Tocopilla main shock (magenta dashed line). An orange dashed line indicates the time of occurrence of a $M 7.1$ aftershock (2007-12-16) 50 km south of the main shock epicentre.
Figure S6. Cumulative number of $\mathrm{P}$ (red curve) and $\mathrm{S}$ (blue curve) phases predicted by DeepPhasePick $\left(p b_{-} P_{-} t h 1=p b_{-} S_{-} t h 1=\right.$ 0.98 ), and triggered detections by the STA/LTA algorithm (green curve) on continuous seismic data recorded by the stations with available data in northern Chile. Last subplot shows the combined results for all the stations in the network. Cumulative curves are plotted for the time interval between two months before and two months after the 2014 M8.1 Iquique main shock (magenta dashed line). Two additional orange dashed lines indicate the time of occurrence of the M6.7 foreshock (2014-03-16) and the M7.6 aftershock (2014-04-03) in the Iquique sequence.

Figure S7. Cumulative number of $\mathrm{P}$ (red curve) and S (blue curve) phases predicted by DeepPhasePick $\left(p b_{\_} P_{-} t h 1=p b_{-} S_{-} t h 1=\right.$ 0.98 ), and triggered detections by the STA/LTA algorithm (green curve) on continuous seismic data recorded by the stations with available data in Albania. Cumulative curves are plotted for the time interval between 2019-12-19 and 2020-02-24, during the aftershock sequence of the 2019 M6.4 Albania earthquake. First 15 stations in the network are shown here.

Figure S8. Similar to Fig. S7, showing results from remaining stations in the Albanian network. Last subplot shows the combined results for all the stations in the network.

Figure S9. Similar to Fig. 21, for detected event e7 in Fig. 20. Pick statistics for this event are reported in Table S3.

Figure S10. Similar to Fig. 21, for detected event e8 in Fig. 20. Pick statistics for this event are reported in Table S4.

Figure S11. Results from the comparison of testing DeepPhasePick and EQTransformer models on the test set including all the correctly classified $P$ - and $S$-phase samples (1430 $P$-phase and $1423 S$-phase samples) from the phase detection evaluation in this work (Fig. 7). (a and b) Time residuals distribution for $P$ - and $S$-phase samples (as in Figs $11 \mathrm{c}$ and 12c) obtained from the preliminary picks computed in the phase detection stage of DeepPhasePick. (c and d) Time residuals from the refined picks estimated after the phase picking stage of DeepPhasePick. (e and f) Time residuals obtained from applying EQTransformer model. Reported mean $(\mu)$, standard deviation $(\sigma)$ and mean absolute error (MAE) are calculated on these residual distributions $(\mid$ tres $\mid \leq 0.8)$.

Figure S12. Results from the comparison of testing DeepPhasePick and EQTransformer models on 1000 randomly selected event samples (1000 $P$-phase and $1000 S$-phase samples) from the STEAD test set. (a and b) Time residuals distribution for $P$ - and $S$-phase samples (as in Figs 11c and 12c) obtained from the refined picks estimated after the phase picking stage of DeepPhasePick. (c and d) Time residuals obtained from applying EQTransformer model. Reported mean $(\mu)$, standard deviation $(\sigma)$ and mean absolute error (MAE) are calculated on these residual distributions $(\mid$ tres $\mid \leq 0.8)$.

Please note: Oxford University Press is not responsible for the content or functionality of any supporting materials supplied by the authors. Any queries (other than missing material) should be directed to the corresponding author for the paper. 